\title{
Un établissement rural gallo-romain sur la commune de Plouër-sur-Rance (Côtes-d'Armor)
}

Romuald Ferrette et Laurent Aubry

\section{OpenEdition}

12 Journals

Édition électronique

URL : http://journals.openedition.org/rao/333

DOI : $10.4000 /$ rao.333

ISBN : 978-2-7535-1607-6

ISSN : $1775-3732$

Éditeur

Presses universitaires de Rennes

Édition imprimée

Date de publication : 30 décembre 2007

ISBN : 978-2-7535-0574-2

ISSN : 0767-709X

Référence électronique

Romuald Ferrette et Laurent Aubry, « Un établissement rural gallo-romain sur la commune de Plouërsur-Rance (Côtes-d'Armor) », Revue archéologique de l'Ouest [En ligne], 24 | 2007, mis en ligne le 03 mai 2010, consulté le 03 décembre 2020. URL : http://journals.openedition.org/rao/333 ; DOI : https:// doi.org/10.4000/rao.333 


\title{
Un établissement rural gallo-romain sur la commune de Plouër-sur-Rance (Côtes-d'Armor)
}

\author{
Romuald Ferrette* \\ avec la collaboration de Laurent AUBRY*
}

Résumé : Une fouille préventive effectuée à Plouër-sur-Rance au lieu-dit "La Mézeray » a permis la découverte d'un bâtiment gallo-romain à la fonction originale mais d'interprétation délicate. Construit avec des matériaux de récupération, issus pour la plupart d'un édifice thermal, il a connu deux grandes phases d'extension. L'existence de bassins et d'une évacuation maçonnée importante suggère qu'on y pratiquait une activité liée à l'eau, et ce quelle que soit la période. L'hypothèse d'un établissement artisanal est la piste privilégiée, d'après l'analyse de ses composantes. Malgré l'absence d'indice concret, il pourrait, par comparaison avec les ateliers de Pompéi ou d'Ostie, être lié à la transformation de la laine. Cette construction pourrait alors s'intégrer dans un complexe plus vaste et - pourquoi pas? -, refléter une partie de la production d'un grand domaine, posant ainsi la question des relations entre ville et campagne.

\begin{abstract}
A rural Gallo-Roman farmstead on the commune of Plouër-sur-Rance (Côtes-d'Armor)
A salvage excavation undertaken at "La Mézeray", Plouër-sur-Rance, revealed a Gallo-Roman building, of distinctive character but uncertain interpretation. Built with recycled materials, most of them from an earlier unlocated thermal building, it has two main extension phases, unfortunately impossible to date precisely, the building being presumably still in use at the transition of the $2^{\text {nd }}-3^{\text {rd }}$ centuries $A D$. The presence of tanks and of a major drainage system suggest some water-linked activity, whatever the period. The workshop hypothesis is suggested from analysis of the building's structure. Despite the absence of solid evidence, comparison with workshops from Pompei or Ostia suggest it could be linked to wool processing. This building could then be part of a larger complex and perhaps have a role in the production activity of some great estate, thus posing the question of town/country relationships in this region.
\end{abstract}

Mots clés : Côtes-d'Armor, Coriosolites, artisanat, gallo-romain, hydraulique, foulons.

Key words: Côtes-d'Armor, Coriosolitae, crafts, Gallo-Roman, hydraulics, fulling.

Le site gallo-romain de "La Mézeray " (parcelle ZA 86, cadastre 1989) est situé sur la commune de Plouër-surRance, à l'extrémité est du département des Côtes-d'Armor. Implanté sur la rive gauche de La Rance, il appartient administrativement à la civitas des Coriosolites dont le cheflieu, Fanum Martis, est l'actuel Corseul (fig. 1). Il se trouve également non loin d'Alet, ville importante durant le BasEmpire, et surtout à $10 \mathrm{~km}$ de l'agglomération portuaire de
Taden, installée à l'ancien point de rupture de charge de la Rance (Langouët 1985; 2004).

Sa découverte fortuite est intervenue au mois de septembre 2002 à la suite des travaux de terrassements routiers de la Départementale 766. Alerté, le Service Régional de l'Archéologie a prescrit une fouille préventive qui s'est déroulée du 12 novembre au 20 décembre 2002, sous la direction de L. Aubry, assisté de trois archéologues ${ }^{1}$.

* INRAP Grand-Ouest, 37 rue du Bignon, CS 67737, Cesson-Sévigné.

1. Aubry et al., 2002. L'équipe de l'INRAP était composée, outre du responsable, de R. Ferrette, E. Gaumé, J.-F. Royer et F. Le Boulanger. La couverture photographique a été assurée par H. Paitier et L. Aubry. Les relevés topographiques ont été réalisés par V. Pommier et la DAO par S. Jean. Nous remercions P. Borgard (Centre Camille-Julian, Aix-en-Provence) pour son aide et la transmission de documents. 


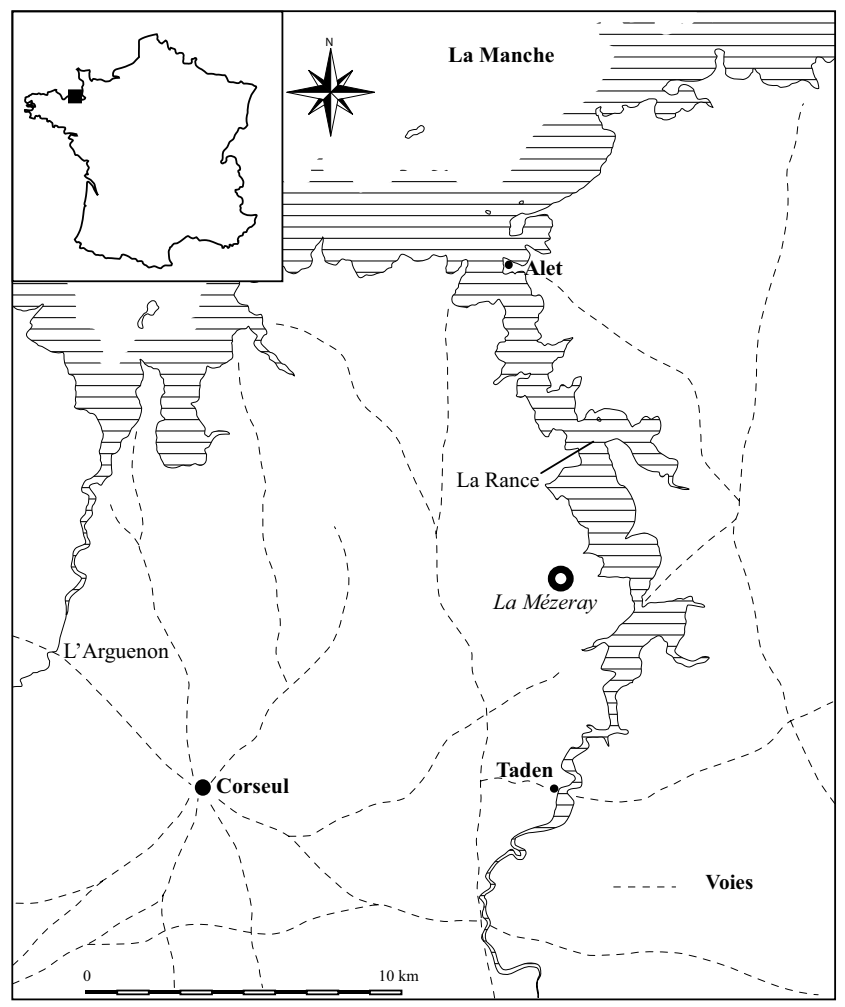

Figure 1 : Localisation du site de " La Mézeray " près de l'embouchure de la Rance (DAO de l'auteur).

Figure 1: Location of the "La Mézeray" site, near the mouth of the river Rance.

\section{L'ENVIRONNEMENT DU SITE}

\section{La topographie et la géologie}

La construction est installée sur le versant nord-est d'un vallon au relief peu prononcé, au fond duquel serpente le ruisseau de la Houssaye. Ce dernier capte sur son parcours de nombreux rus, ainsi qu'une grande partie des eaux de ruissellement des terres environnantes. Le secteur est également riche en sources et l'une d'entre elles a d'ailleurs été reconnue en limite sud de l'intervention, à une vingtaine de mètres de l'édifice. Son excédent a alimenté un ruisselet, aujourd'hui totalement comblé, qui cheminait à travers le site selon un axe est-ouest. Un dépôt de tourbe, observé dans la partie basse de l'intervention, correspond à une baisse de la dynamique de ce cours d'eau bien après la destruction du bâtiment.

Les formations superficielles qui scellaient le gisement étaient totalement bouleversées du fait des terrassements mécaniques qui ont précédé sa découverte. Leur nature et leur ampleur sont donc difficilement estimables. Compte tenu du contexte topographique, la présence de colluvions était fort probable et les parcelles adjacentes montrent d'ailleurs une couche limoneuse d'une épaisseur d'une quarantaine de centimètres. Dans la partie haute de l'intervention, les schistes briovériens plus ou moins altérés affleurent, puis disparaissent au contact du bâtiment pour laisser place à un pseudo-gley gris orangé et feuilleté, dans lequel sont installées les différentes maçonneries. Dans la partie basse de la parcelle, cette formation devient plus sableuse, peut-être en raison de la présence de l'ancien ruisseau.

\section{Les contraintes techniques et scientifiques}

Lédifice, dans son extension maximale, s'inscrit dans un rectangle mesurant $19 \mathrm{~m}$ de long pour une largeur proche de $11 \mathrm{~m}$ et son orientation obéit à la pente du terrain (fig. 2). Il se caractérise par une succession de salles de petites tailles, précédées au nord-est par un espace cloisonné. Une imposante canalisation longe son pignon nord-ouest, ainsi que l'arrière. Aucun aménagement spécifique n’a été repéré dans sa périphérie : seuls quelques creusements, principalement des fossés, ont été découverts à l'issue du décapage. Leur remplissage superficiel dénote la présence de matériaux antiques, liés sans doute à des démolitions (moellons, fragments de terres cuites).

La stratégie de fouille mise en œuvre a pris en compte deux paramètres : l'état plus ou moins dégradé de l'édifice et les conditions pluviométriques lors de l'intervention (fig. 3). Dans un premier temps, la partie haute de la construction a été étudiée car elle était la mieux conservée et la plus à même de nous fournir le maximum d'informations sur la fonction et le phasage de l'édifice. Cette zone était en outre préservée des intempéries, le secteur le plus proche du talweg, le plus arasé, étant en revanche fréquemment inondé. Nous sommes parfaitement conscients que les observations y ont été incomplètes.

A priori, le plan du bâtiment est complet. Nonobstant une destruction partielle de la zone nord-est par les terrassements routiers, il peut en effet être restitué dans ses grandes lignes (fig. 2). Il est ainsi peu probable que des maçonneries se soient développées au-delà du mur M 2043. Au nord, M 2015 marque l'extension maximale du bâti. À l'ouest, les murs M 2010 et 2038 semblent aussi constituer une limite fiable. En revanche, l'existence d'appentis annexes totalement érodés ne peut être écartée.

Plusieurs difficultés sont apparues lors de l'analyse de l'évolution structurelle du bâtiment, qui ont une incidence sur l'interprétation de sa fonction. Dans la zone affectée par les terrassements, les maçonneries ont subi de nombreux dégâts et la disparition de niveaux de remblais ou de sols encore en place ne peut être totalement exclue. Une deuxième difficulté résulte du démantèlement que l'édifice a connu après son abandon. De nombreuses maçonneries 


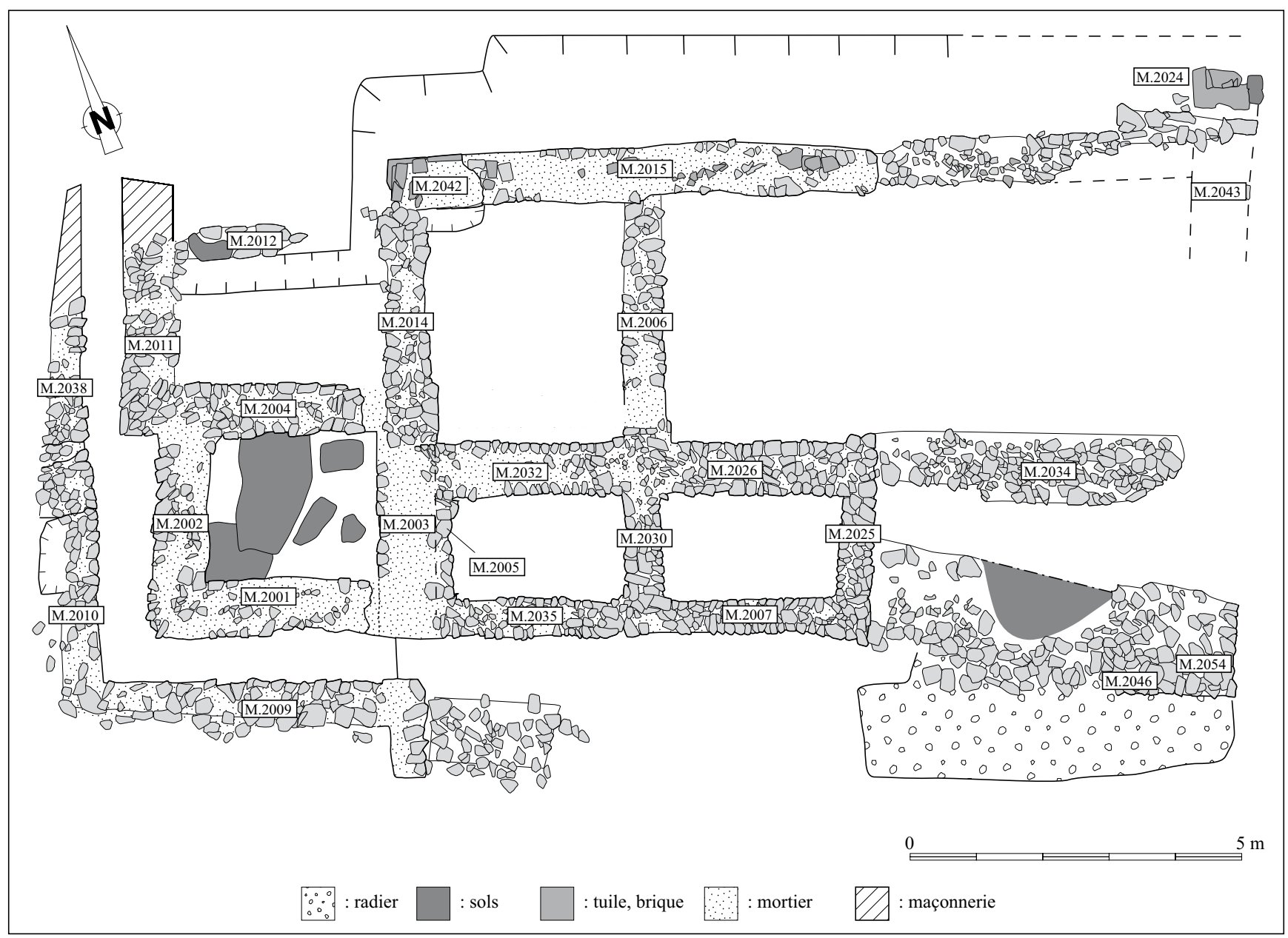

Figure $2:$ "La Mézeray " : plan général du bâtiment et nomenclature des murs identifiés (DAO S. Jean - INRAP). Figure 2: General plan of the "La Mézeray" building and nomenclature of its identified walls.

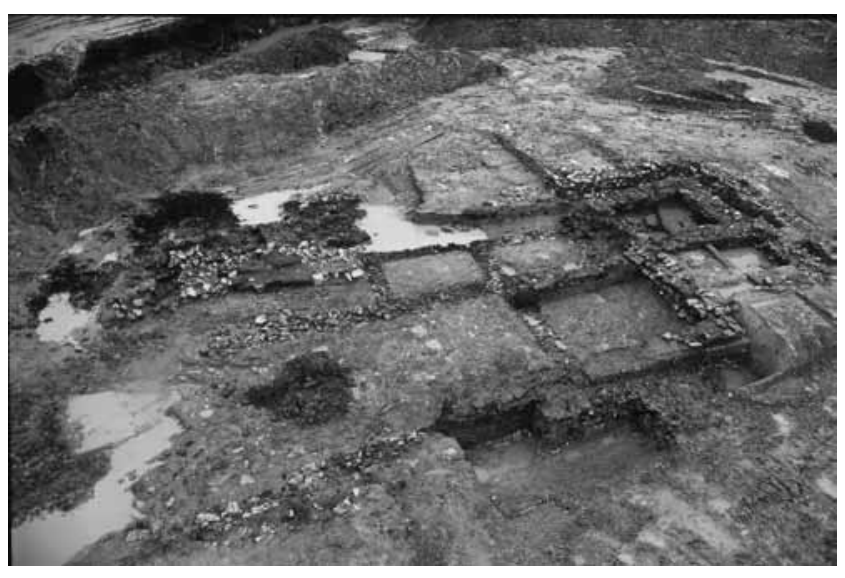

Figure 3 : Vue générale du site depuis le sud-ouest (cl. H. Paitier - INRAP).

Figure 3: General view of the site, from South-West. avaient été démontées et la plupart des niveaux d'occupation et de sols avaient disparu avant la fouille. Enfin, la rareté du mobilier constitue une dernière contrainte car il est impossible de critiquer la chronologie relative du bâti et d'établir des datations solides.

\section{L'ÉVOLUTION DU SITE}

Malgré ces difficultés, quatre grandes phases ont été mises en évidence. Les trois premières correspondent à des périodes de construction et d'extension du bâti, la dernière rendant compte du démantèlement et de la ruine définitive de l'édifice.

\section{Phase 1 : la construction initiale}

Les données disponibles pour cette première phase sont assez lacunaires, en raison de l'arasement des vestiges mais 
aussi des transformations ultérieures. Le bâtiment comprend au moins deux pièces, précédées d'un grand espace cloisonné par une maçonnerie. Une dernière salle peut être aussi envisagée dans la partie nord-ouest (fig. 4).

\section{Les pièces 1 et 2}

Ces deux pièces, au plan rectangulaire similaire, possèdent une longueur de 2,70 m et une largeur de 1,56 m. Leur conservation est très médiocre : tous les niveaux contemporains de leur construction ou de leur fonctionnement ont été démontés après l'abandon du bâtiment. Les murs sont arasés au niveau du substrat glaiseux, qui prend une coloration bleue en raison d'un niveau de tourbe (US 1001) qui couvre l'intégralité de ce secteur et témoigne d'une stagnation d'eau et du dépôt progressif de végétaux. Ce dépôt tourbeux, postérieur à la phase de démontage, a livré une anse d'objet indéterminé en bronze, apparemment antique, figurant un dauphin stylisé (fig. 5).

La technique de construction de ces deux premiers ensembles est aisément restituable. Les ouvriers ont d'abord décaissé le sol naturel à leur futur emplacement afin de réaliser une esplanade, puis creusé les différentes tranchées de fondation des murs. Ces dernières sont appareillées en micaschiste et assises sur des blocs de quartz. Aucune trace de mortier n'a été mise en évidence, ce qui peut résulter des conditions particulières de conservation. Le blocage comprend de la pierraille de micaschiste, associée parfois à des moellons récupérés. Les murs M 2006 et 2007/2035 offrent une largeur similaire, d'une soixantaine de centimètres. En revanche, M 2026/2032 atteint $80 \mathrm{~cm}$. Les murs pignons sont moins larges, de l'ordre d'une cinquantaine de centimètres.

\section{Les pièces 3 et 4}

L'existence de ces deux espaces, aux limites incertaines, est déduite des maçonneries M 2015, 2030 et 2043, qui se développent au nord des précédentes (fig. 4).

Le mur 2030, situé dans le prolongement de M 2006, présente une largeur de $60 \mathrm{~cm}$ et a été épierré jusqu'au niveau du substrat. Chaîné avec M 2026 et M 2032, il est parementé de blocs de micaschiste et se développe sur une longueur de 3,60 m. Malgré l'absence d'imbrication avec M 2015, ces

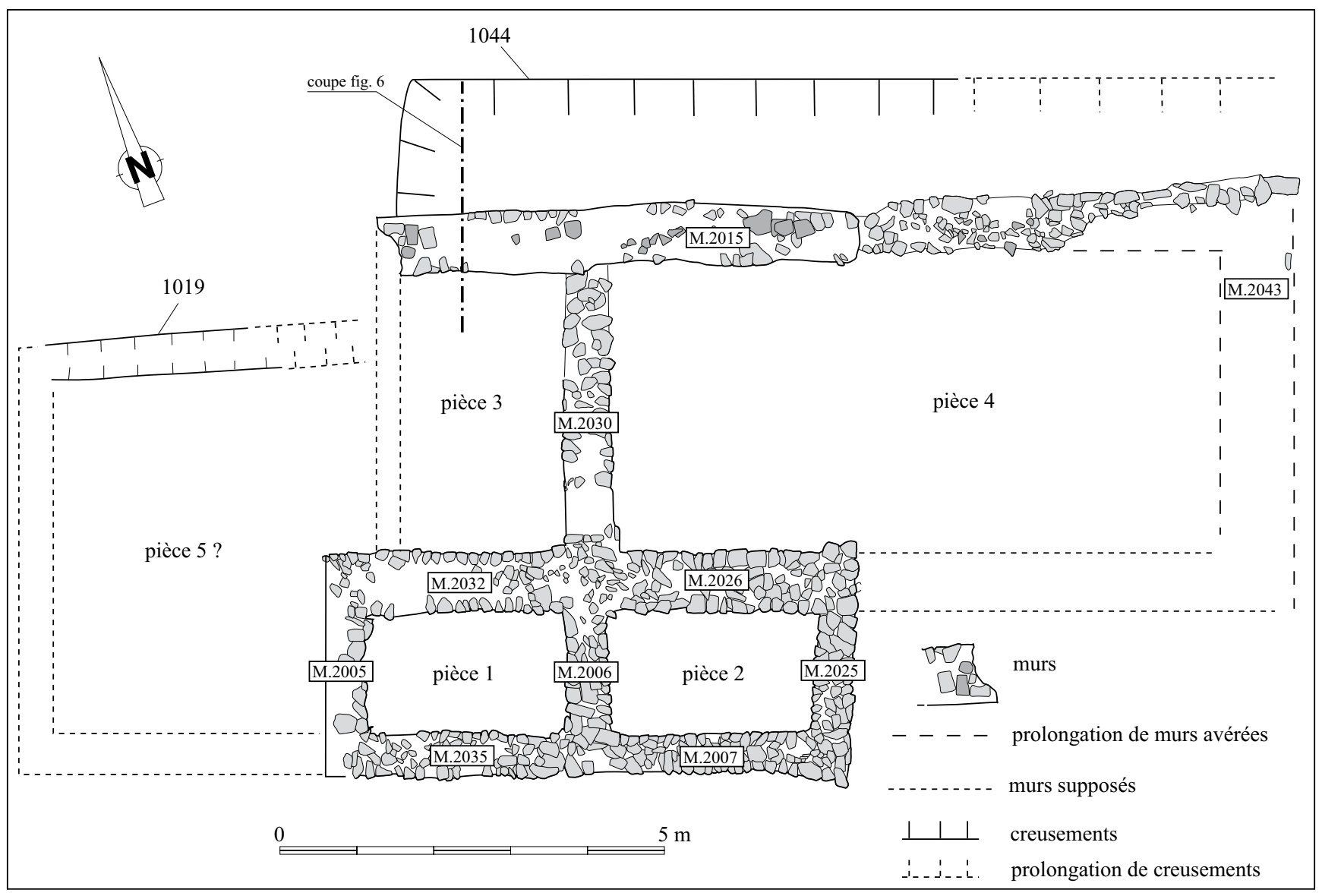

Figure 4 : "La Mézeray " : plan des vestiges de la phase 1 (DAO S. Jean - INRAP).

Figure 4: Plan of phase 1 remains. 


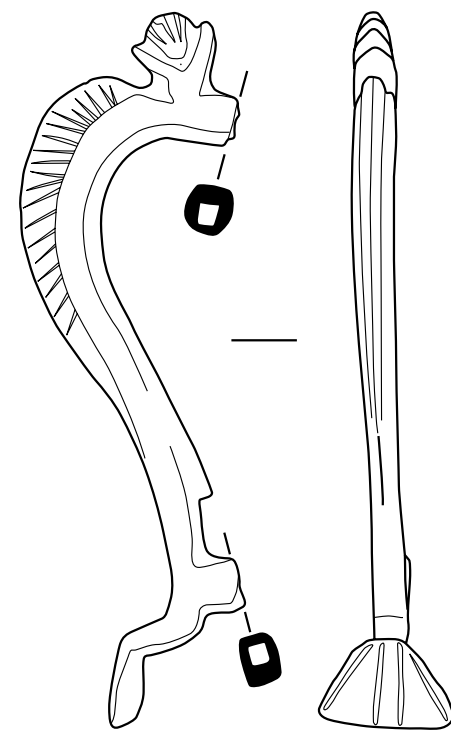

0

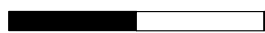

Figure 5 : Anse en métal cuivreux découverte dans la couche 1001 de la phase 4, pièce 1 (DAO S. Jean - INRAP).

Figure 5: Copper alloy handle from layer 1001; phase 4, room 1.

deux murs doivent être considérés comme contemporains, M 2030 étant simplement un refend.

M 2015 se déploie sur une longueur de $11 \mathrm{~m}$ environ. Son extrémité ouest est soulignée de pierres d'angle en micaschiste ou plus exceptionnellement en granite (1 exemple), dont les dimensions atteignent $30 \mathrm{~cm}$ de long pour une hauteur de $6 \mathrm{~cm}$. Il est assez bien conservé dans sa partie ouest, sur une longueur de $6 \mathrm{~m}$ environ. En revanche, sa portion orientale a subi d'importants dommages consécutifs aux terrassements routiers : seule la dernière assise, permettant de suivre son tracé, est conservée. Ce mur est également bordé au nord d'un creusement important (US 1044).

Large de $80 \mathrm{~cm}$ en fondation, M 2015 doit être considéré comme un mur porteur venant clore au nord le bâtiment. Son arasement, large seulement de $75 \mathrm{~cm}$, est marqué d'une assise de briques qui pourrait indiquer la limite de son soubassement. Son appareillage de moellons de micaschiste est jointoyé au mortier de chaux et soigneusement agencé. Le blocage est réalisé à l'aide de micaschiste, de fragments de tuiles et de rares blocs de quartz. Sur sa face nord, il est parementé jusqu'au substrat, c'est-à-dire jusqu'au fond du creusement 1044. Les assises de moellons reposent alors sur un rang de blocs de quartz liés au mortier. Sa mise en œuvre peut facilement être restituée. Le sol naturel a été décaissé sur une largeur beaucoup plus importante que l'emprise du mur lui-même. Cette largeur englobe M 2015 à laquelle s'ajoute une bande de 1,80 m au maximum, qui correspond au creusement 1044. Dès lors, on peut envisager la réalisation de M 2015 depuis le nord, dans une tranchée de fondation large et ouverte, sa face sud venant s'adosser au sol naturel.

En raison des terrassements routiers, la maçonnerie M 2043, qui ferme la pièce 4 à l'est, a été observée sur une longueur inférieure à $1 \mathrm{~m}$ et sa largeur est évaluée à $80 / 90 \mathrm{~cm}$ environ. Seuls demeurent quelques blocs de quartz qui viennent buter contre M 2015.

Le creusement 1044 obéit quant à lui à la topographie et affecte une pente est-ouest. L'interprétation comme un fossé comblé volontairement et destiné à drainer les remontées d'eau par capillarité vers le talweg semble vraisemblable d'après le type de remplissage (fig. 6, US 1054).

Aucun niveau de circulation contemporain de cette première phase n'est conservé à l'emplacement des salles 3 et 4 . Seule subsiste une couche (US 1056) formée de nombreux éléments en terre cuite (briques, tuiles...) et de micaschiste, qui repose directement sur le pseudo-gley. Ce niveau, qui s'appuie sur M 2015, est un remblai drainant.

\section{Une salle occidentale? La pièce 5}

L'existence de cette pièce demeure plus qu'hypothétique. Ses vestiges potentiels sont très ténus et se résument à un creusement est-ouest qui s'étire sur plus de $2 \mathrm{~m}$, mais son développement total n'est pas connu en raison des aménagements postérieurs (fig. 4, US 1019). Possédant une profondeur de $25 \mathrm{~cm}$ pour une ouverture maximale de $50 \mathrm{~cm}$, ce creusement peut correspondre à un solin ou à une fondation entièrement récupérée. Dans cette optique, la question de son retour vers le sud reste posée.

\section{La phase 2 : une première extension du bâti}

Cette seconde période marque une première extension du bâti, alors que les salles antérieures sont conservées. Les principales modifications concernent l'apparition d'un bassin (pièce 6) à l'ouest de la pièce 1 , ainsi que la création d'une canalisation maçonnée (US 2023) le long de ce nouvel espace (fig. 7 et 8 ).

\section{L'apparition d'un bassin : la salle 6}

Cette salle mesure, hors œuvre, $4 \mathrm{~m}$ de long pour 3,6 m de large environ et s'installerait pour partie à l'emplacement de la pièce 5 de la phase antérieure (fig. 8). Sa superficie interne est légèrement supérieure à $4 \mathrm{~m}^{2}(240 \times 210 \mathrm{~cm})$ et ses murs conservent une élévation de 40 à $45 \mathrm{~cm}$ depuis la surface de son sol bétonné (US 2016). 


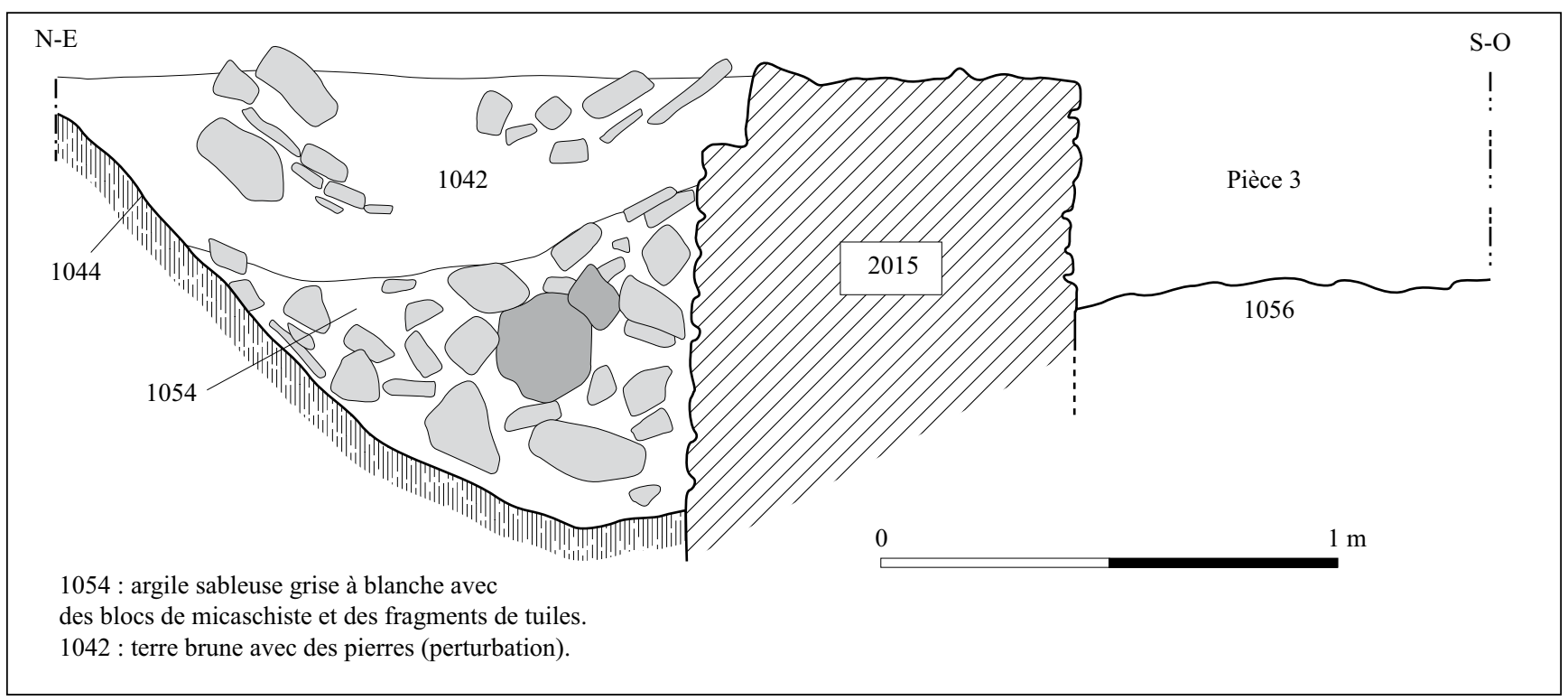

Figure 6 : Coupe stratigraphique du fossé drainant 1044 (DAO S. Jean - INRAP). Figure 6: Stratigraphic section of drainage ditch 1044.

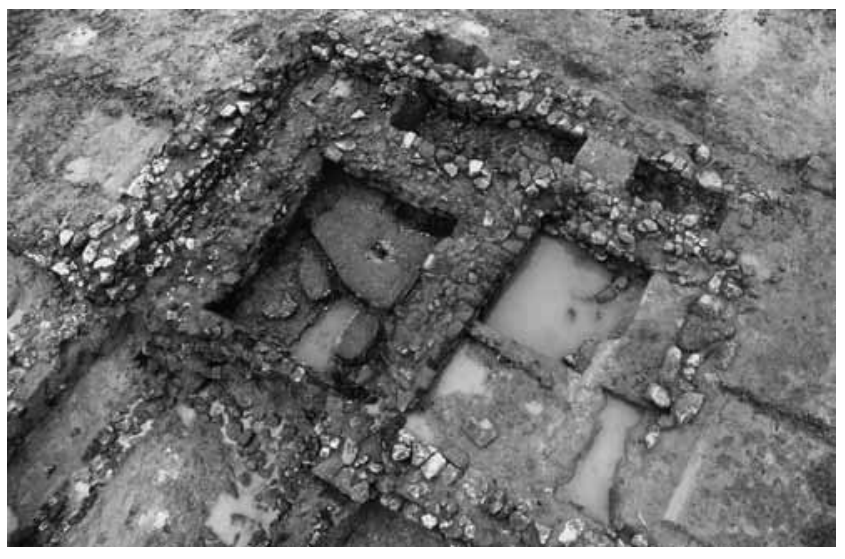

Figure 7 : Vue de la pièce 6 bordée par la canalisation 2023 (cl. H. Paitier - INRAP).

Figure 7: View of room 6 bordered by channel 2023.

Les maçonneries 2001 à 2004, qui la délimitent, ont une largeur de $80 \mathrm{~cm}$ et sont parementées de moellons de micaschiste et de gneiss de modules variables. L'emploi de quartz blanc demeure anecdotique. Le blocage comprend du micaschiste et du gneiss, le tout jointoyé au mortier de chaux de couleur crème. M 2001 et M 2002 sont parementés jusqu’au niveau du radier de fondation de la canalisation 2023, au contraire de M 2004, profondément ancré dans le sol naturel en raison d'un fossé le longeant (US 1028). M 2003, qui ne dispose pas d'une fondation puissante, s'appuie contre M 2005 de la salle 1. C'est d'ailleurs sans doute cette mise en œuvre spécifique qui justifie le renfort, à l'aide de gros blocs imbriqués, de l'angle M 2001/2003. Ajoutons que les parements externes de M 2001 et M 2002 sont enduits d'une couche de mortier de tuileau (US 2018), en raison de la présence de la canalisation 2023. Le parement extérieur de M 2003 est aussi enduit de mortier hydrofuge dans sa partie supérieure, contrairement au parement nord de M 2004 qui en est dépourvu. Aucune structure liée à l'eau n'a donc dû exister en avant de la pièce 6 durant cette seconde période. Précisons enfin que toutes les maçonneries disposaient d'un revêtement de mortier de tuileau sur leur face interne, ce qui se comprend par la fonction de la salle 6 .

Tout indique que cette salle était au départ un bassin dont l'arrivée d'eau paraît matérialisée par un aménagement dans l'angle interne nord-est de la salle. Il existe en effet, au niveau de la jonction entre M 2003 et M 2004, un vide de 20 x $20 \mathrm{~cm}$ environ (US 2037), tapissé de mortier hydrofuge, qui pourrait correspondre à une arrivée d'eau verticale. La base de 2037 se trouvant à $17 \mathrm{~cm}$ au-dessus du sol 2016, le bassin devait être peu profond. L'évacuation du bassin est quant à elle constituée d'une ouverture dans la partie médiane du mur 2001 (US 2017), qui débouche directement dans la canalisation 2023. Il s'agit d'une conduite en tubuli de $15 \mathrm{x}$ $15 \mathrm{~cm}$ dans laquelle devait courir un tuyau de plomb, protégé ainsi d'un écrasement. Lorsque le bassin était utilisé, cet exutoire devait être obturé à l'aide d'un bouchon de bois.

Le sol de la pièce 6 est formé d'un niveau de mortier de tuileau dont l'épaisseur est estimée à $2 \mathrm{~cm}$, son épiderme ayant été préservé des outrages du temps sur une très faible surface (fig. 9, US 2016). Les altitudes relevées prouvent cependant qu'une pente volontaire nord-sud a été aménagée afin de faciliter l'écoulement. Ce sol repose sur un rudus en 


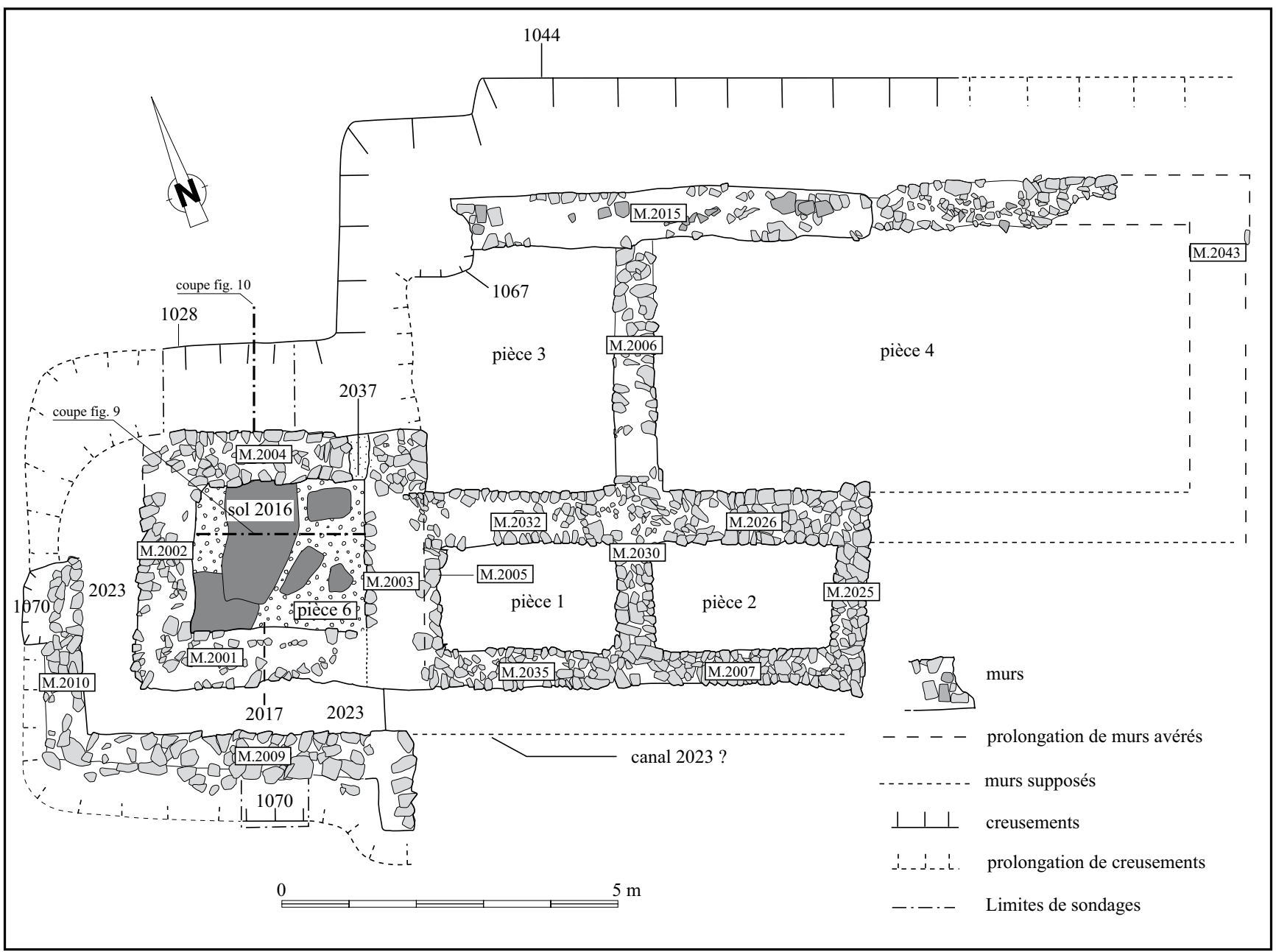

Figure 8 : «La Mézeray» : plan des vestiges de la phase 2 (DAO S. Jean - INRAP). Figure 8: Plan of remains from phase 2.

béton de tuileau épais d'une dizaine de centimètres, comportant de la pierraille et des nodules calcaires (US 2020). Il a en partie été démonté, sans doute lors de la restructuration de la pièce ou sinon après son abandon définitif. Cette semelle repose sur un radier (US 2021), agencé avec des blocs de micaschiste, de quartz et de rares fragments de tuiles, le tout lié à l'argile. Il repose directement sur le substrat, dont le sommet se place aux alentours de 72, $80 \mathrm{~m}$ NGF, c'est-à-dire à une altitude supérieure de 50 à $60 \mathrm{~cm}$ à celle relevée à l'emplacement des salles 1 et 2 , laissant supposer que nous sommes en présence d'espaces aménagés en terrasses successives (cf. infra, fig. 13).

Le radier 2021 se développe jusqu'aux murs M 2001 à 2004, ce qui n'est pas le cas du rudus. Il existe en effet, entre sa terminaison et les différentes maçonneries, un espace d'environ $10 \mathrm{~cm}$ (fig. 9, US 2022), comblé par de l'argile sableuse (US 1074) elle-même surmontée d'un cordon de mortier hydrofuge épais de $3 \mathrm{~cm}$ (US 2016bis). L'hypothèse que cet emplacement marque un équipement spécifique le long des différentes maçonneries est plus que probable. Il pourrait s'agir d'un emmarchement démonté lors de l'ultime phase d'agrandissement de la construction.

\section{Le canal maçonné}

Le second élément majeur est l'apparition d'un canal maçonné (US 2023). On ignore cependant s'il s'agit d'une création nouvelle ou s'il se substitue à une conduite antérieure, dont aucun vestige ne serait conservé. Durant la phase 2, son emprise est délimitée d'une part par les murs 2001 et 2002 de la salle 6, d'autre part par M 2009 et M 2010 (fig. 8). Le développement de ce canal en amont de la pièce 6 et le long des pièces 1 et 2 n'est pas connu.

La maçonnerie 2009 présente une longueur de $5,25 \mathrm{~m}$ environ, avant d'opérer un retour dans l'axe de M 2003. Sa largeur oscille entre 40 et $60 \mathrm{~cm}$ et elle comporte une élévation de $70 \mathrm{~cm}$ par rapport au sommet du radier du canal. 


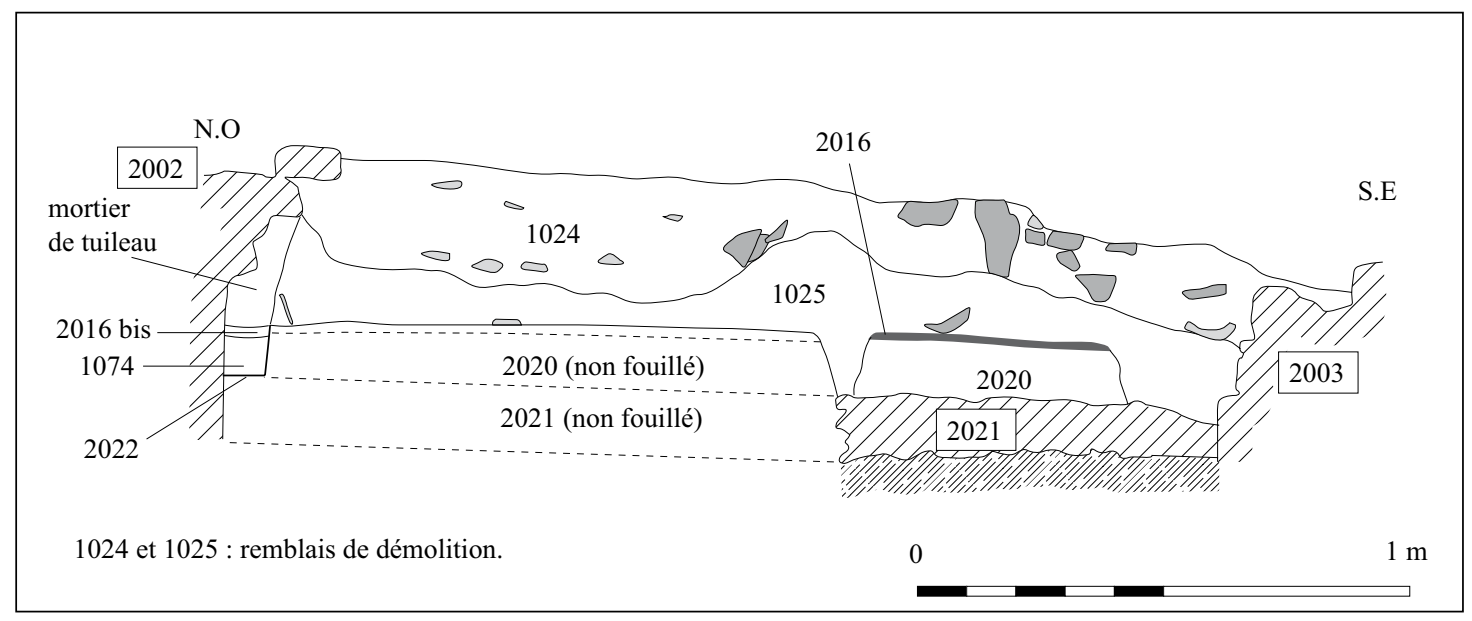

Figure 9 : Coupe stratigraphique de la salle 6 (DAO S. Jean - INRAP).

Figure 9: Stratigraphic section of room 6.

Son appareillage est constitué de quartz blancs et de rares blocs de micaschiste ( $20 \times 15 \mathrm{~cm}$ en moyenne), jointoyés au mortier de chaux et grossièrement équarris.

À l'ouest, M 2010 court sur une longueur de 3 m environ, sa terminaison nord étant incertaine du fait des dernières transformations. À l'image de M 2009, il est réalisé à l'aide de moellons de quartz dont les quatre assises conservées forment un parement à l'intérieur du canal (50 cm d'élévation). Le blocage de ces deux maçonneries comprend des éléments de quartz liés au mortier de chaux. Aucune trace de revêtement de tuileau n'a été décelée sur leurs parements.

Le canal proprement dit (US 2023) est large de 75 à $80 \mathrm{~cm}$. Malgré un état de conservation assez déplorable, l'examen de ses différents composants démontre une mise en œuvre très soignée. Un premier niveau est constitué d'un puissant radier (US 2019) s'appuyant sur les murs qui le délimitent. Posé directement sur le substrat, ce radier comprend du quartz et du micaschiste et présente un pendage qui obéit à la topographie. Aucun aménagement n'a été mis en évidence à son sommet, hormis dans l'angle sud-ouest de la conduite. Le radier est alors surmonté de trois rangées de terres cuites architecturales. L'assise affleurante est formée de briques rectangulaires associées à des tuiles à rebord retournées. L'assise intermédiaire est réalisée à l'aide de fragments de tegulae qui ont été soumises à une forte chaleur, ce qui plaide en faveur d'éléments récupérés. Agglomérés à l'argile, ces lits de terres cuites, dont on ignore s'ils sont tous contemporains où s'ils correspondent pour les deux derniers à des réfections du canal d'écoulement, devaient être surmontés d'une chape de mortier hydrofuge, qui marquait le véritable niveau de circulation de l'eau.

\section{Le fossé périphérique 1028/1070}

Les fossés 1028 et 1070 forment une seule et même structure qui vient se raccorder à la tranchée 1044 par l'intermédiaire d'un creusement (US 1067) lui-même recoupé par des aménagements postérieurs (fig. 8). Leur rôle, comme le souligne le remplissage de 1028 (fig. 10), était de protéger des infiltrations d'eau les nouvelles maçonneries de la phase 2 .

\section{La phase 3 : d'importants remaniements}

Les ultimes travaux décelables sur ce bâtiment se concrétisent par l'adjonction d'une salle à l'est de la pièce 2 et d'une seconde au nord de la pièce 6 , qui subit elle-même des modifications. Le canal 2023 est prolongé vers le nord et la tranchée 1028/1044/1070 est condamnée (fig. 11).

\section{La pièce 7 : un nouveau bassin?}

Elle forme un rectangle mesurant, hors ouvre, $6 \mathrm{~m}$ de long pour $4 \mathrm{~m}$ de large et est bordée au sud d'un important massif de blocs de quartz (US 1023). Sa conservation est très médiocre car ses vestiges encore en place ont en partie été détruits par les terrassements routiers, de même que son sol (US 2027). Sa limite nord, M 2034, est un radier de blocs de quartz associés à du micaschiste, qui vient s'accoler à M 2025. Cette maçonnerie possède une largeur de $80 \mathrm{~cm}$ dans sa partie haute, à l'instar de M 2036/2032 des pièces 1 et 2, ce qui témoigne d'un mur porteur. M 2034 s'élargit ensuite à la hauteur du sol 2027, pour atteindre $1 \mathrm{~m}$ environ.

Le mur sud de la pièce 7 a été reconnu sur $4,5 \mathrm{~m}$ (M 2046) : il est parementé sur près de $2 \mathrm{~m}$, puis un alignement de gros blocs de quartz permet de suivre son tracé qui finit par se perdre. M 2054, qui constitue son retour à l'est, se signale seulement par quelques blocs montés au 


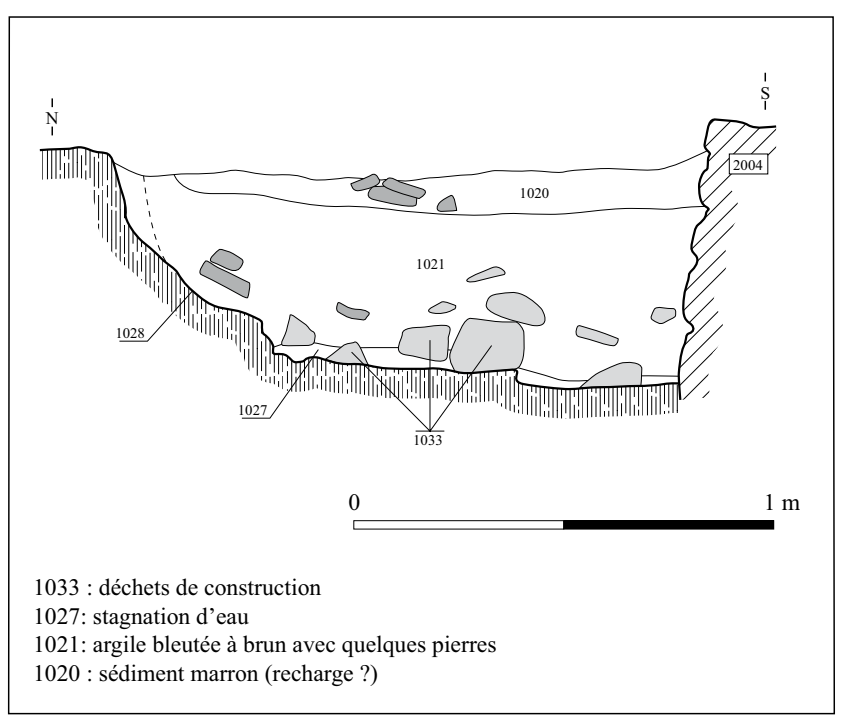

Figure 10 : Coupe stratigraphique du fossé 1028 (DAO S. Jean - INRAP).

Figure 10: Stratigraphic section of ditch 1028. mortier de chaux. Les parements de ces deux maçonneries sont constitués de moellons en micaschiste $(30 \times 25 \mathrm{~cm})$, qui soutiennent un niveau de quartz (US 2008), néanmoins soigneusement agencé au contact du sol 2027.

Ce dernier n'est pas conservé dans son intégralité mais seulement sur une surface en forme d'arc de cercle. Il culmine à 75,58 m NGF mais on ignore s'il s'agit bien de son épiderme. Il est composé d'un lit de mortier de tuileau orangé très altéré par l'humidité, d'une dizaine de centimètres d'épaisseur, qui recouvre un lit de fragments de tuiles et de tubulures posés à plat (US 2049). Cette unité coiffe un second niveau de mortier hydrofuge plus rouge, installé sur le substrat et qui se développe seulement sur le pourtour de la pièce. Il n'est pas possible de préciser s'il correspond aux vestiges d'un premier sol ou si son installation est contemporaine de celle des US 2027 et 2049.

La salle 7 pourrait en tout cas s'apparenter à un bassin, dont les systèmes d'adduction et de vidange ne sont pas connus, du fait de son état de conservation très dégradé.

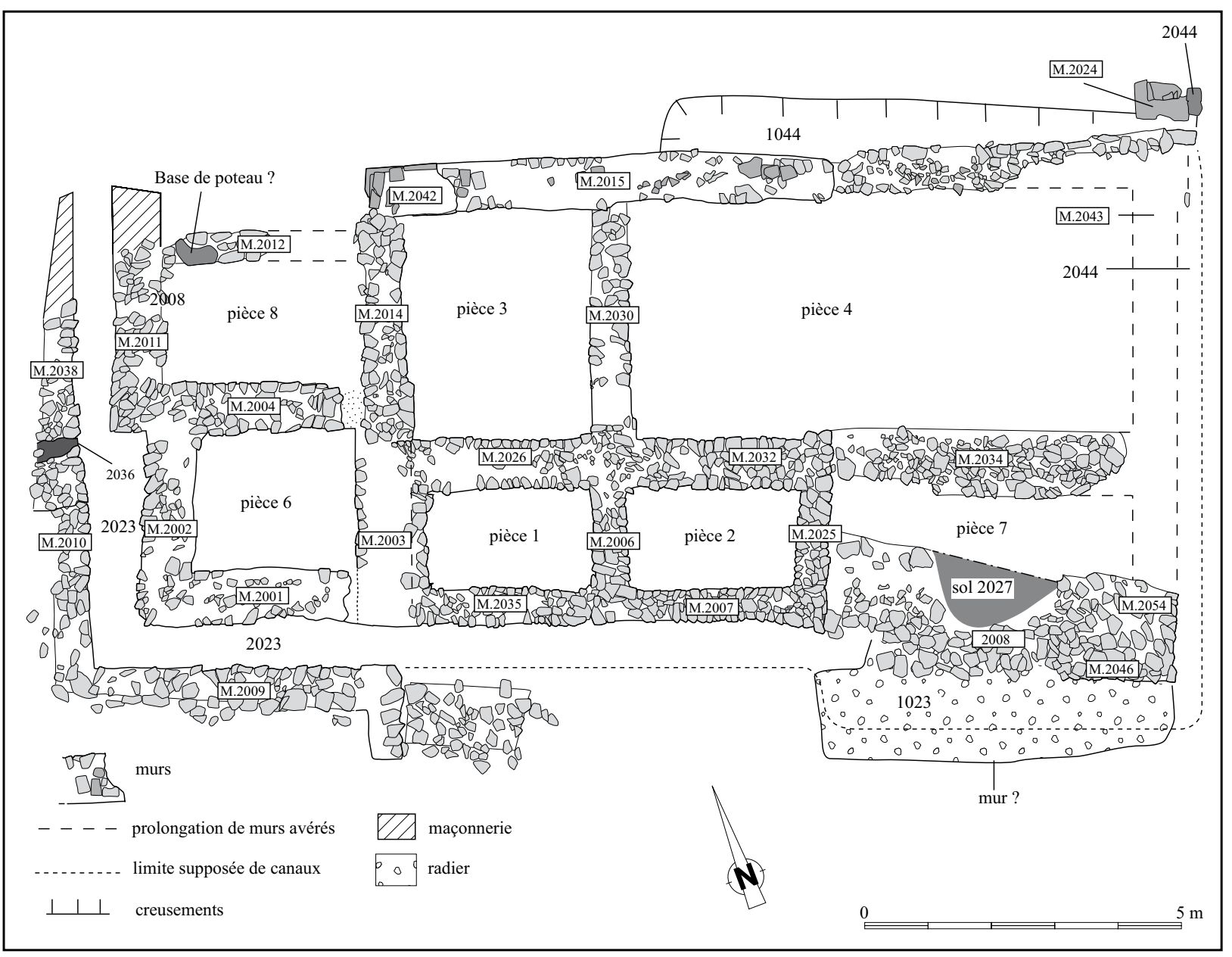

Figure 11 : «La Mézeray » : plan des vestiges de la phase 3 (DAO S. Jean - INRAP).

Figure 11: Plan of remains from phase 3 . 


\section{La prolongation du canal 2023}

Lors de son apparition à la phase 2 , aucun indice pertinent n'autorisait à suivre le tracé de ce canal au nord de la pièce 6 , ce qui n'est désormais plus le cas. Par ailleurs, la création de la salle 7 induit aussi un changement de son cheminement, s'il longeait bien les salles 1 et 2, car il est désormais obligé de contourner ce nouvel espace (fig. 11). Dans la partie nord-ouest du bâtiment, sa prolongation se traduit par la création des murs 2011 et 2038, dont l'écartement est seulement de $65 \mathrm{~cm}$, alors qu'il est de 75 à $80 \mathrm{~cm}$ entre M 2002 et M 2010.

Le mur M. 2038 se développe sur une longueur de $5 \mathrm{~m}$ environ. Il comporte une largeur maximale de $1 \mathrm{~m}$ au niveau de sa jonction avec $M 2010$, qui se réduit à moins de $40 \mathrm{~cm}$ à son extrémité nord mais il est probable que cette dernière ne matérialise pas sa véritable extrémité compte tenu de l'arasement de cette partie du site. M 2038 est agencé à l'aide de blocs de quartz grossièrement équarris qui forment un parement à l'intérieur du canal. Aucune trace de mortier de tuileau n'a été relevée sur celui-ci. Sa jonction avec M 2010 est marquée d'un " coup de sabre " qui illustre parfaitement les modifications apportées lors de cette ultime phase. Un aménagement large de $25 \mathrm{~cm}$ (interruption de la maçonnerie) se dessine aussi en vis-à-vis de l'angle entre M 2011 et M 2002 (fig. 11, US 2036). La possibilité d'une seconde arrivée d'eau débouchant dans le canal, et dont ne subsisterait plus que l'empreinte, n'est pas à exclure.

M 2011, long de 3,5 m et large de $80 \mathrm{~cm}$, est réalisé selon la même technique que le mur précédent. Une bande verticale, se développant le long de son parement et comprenant de nombreuses particules de mortier de tuileau, pourrait à titre d'hypothèse matérialiser les traces d'un revêtement altéré.

Le radier de cette nouvelle portion de la conduite est formé de blocs de quartz blancs liés à l'argile et présente une pente nord-sud. Sa jonction avec le radier de la phase antérieure (US 2019) est soulignée d'un " coup de sabre " qui confirme une reprise.

Comme pour la période antérieure, nous ignorons de quelle manière se développait le canal 2023 le long des pièces 1 et 2 car aucune maçonnerie ne délimite son tracé au sud. La création de la pièce 7 autorise quelques propositions. Cet espace est précédé sur son côté sud d'un important massif de blocs de quartz dont la largeur, $130 \mathrm{~cm}$, peut correspondre d'une part à l'emprise d'une conduite de $80 \mathrm{~cm}$, équivalente à l'intervalle constaté entre M 2001et M 2009, d'autre part à l'emplacement d'un mur d'une cinquantaine de centimètres (fig. 11, US 1023). Dans cette optique, le canal 2023 borderait bien les salles 1 et 2 et formerait un coude au niveau de la salle 7 pour la longer ensuite. L'évacuation de ce bassin se ferait alors sur son côté sud ou est.

\section{La pièce 8 et la maçonnerie 2042}

Ce nouvel espace se développe au nord de la salle 6. Il est limité d'une part par M 2002 et M 2011, d'autre part par M 2014 et M 2012 (fig. 11). Aucun niveau de sol n'est conservé et les murs 2014 et 2012 sont arasés au niveau de leur fondation. M 2012 se développe sur un peu plus de $2 \mathrm{~m}$ de long pour une largeur d'une soixantaine de centimètres. Son radier est formé de quartz mais on observe aussi un fragment de sol en béton de tuileau posé à plat, qui a pu servir d'assise à un poteau. M. 2014, formé d'une superposition de gros blocs de quartz jointoyés au mortier de chaux, vient s'appuyer sur la maçonnerie 2042. Cette dernière, qui constitue le prolongement du mur 2015 apparu lors de la première phase, vient condamner le fossé drainant 1028/1044/1070. M 2042 est constituée à sa base de deux rangs de quartz blanc, surmontés de trois assises de moellons de micaschiste liées au mortier de chaux. Celles-ci sont coiffées de quatre rangs de briques de maçonnerie, mesurant chacune $23 \times 48 \mathrm{~cm}$. Deux nouvelles assises de moellons de micaschiste et de granite et un dernier rang de tuiles, qui marque l'arasement, surmontent l'ensemble.

\section{Autres transformations d'espaces antérieurs}

Cette dernière phase de construction se traduit par un changement de statut du bassin 6 . Son évacuation est obturée à l'aide de mortier de tuileau et l'aménagement 2022, qui courait le long des maçonneries, est démonté. La fonction de cet espace, qui n'est plus un bassin, ne peut être précisée.

Le fossé 1044 connaît également une modification. Une évacuation, sans doute coffrée d'après la découverte de planches de bois, est installée le long de la pièce 4. Elle se termine par une pile de briques maçonnées jointoyées au mortier de chaux, dont huit rangs sont conservés (fig. 11, M 2024). Cette pile est elle-même bordée à l'est par un autre canal dont la fonction n'est pas clairement établie (fig. 11, US 2044). Très dégradé, il est formé d'un radier surmonté d'une chape de béton de tuileau. Il constitue probablement le pendant du canal 2023 et tout deux devaient rejoindre un exutoire aujourd'hui disparu.

\section{Phase 4 : la ruine de l'édifice}

Le bâtiment, encore visible dans le paysage, sert ensuite de carrière. Plusieurs maçonneries sont démontées, à l'instar de celles des salles 1 et 2 , alors que d'autres semblent subsister, à l'image des murs de la salle 6 . L'emplacement des salles 1 et 2 paraît en outre avoir été soigneusement nettoyé, compte tenu de l'absence de niveau en place. Curieusement, le mur 2015 ne semble pas affecté par ces travaux, au moins en profondeur, alors que M 2030 est épierré. Cette zone est alors envahie par un niveau de tourbe (US 1001) et la partie 
basse de la construction devient une zone humide, peut-être une mare dont la création a pu être intentionnelle. Les murs de la salle 6 finissent par verser, puis le site est recouvert de colluvions. Quelques témoins diffus soulignent pourtant le maintien d'une fréquentation du secteur. L'existence d'une fosse (US 1015), qui a livré de nombreuses scories coulées, témoigne ainsi d'une activité de métallurgie à une date inconnue, mais postérieure à la ruine totale de l'établissement d'après la stratigraphie.

\section{LE MOBILIER ET LE PROBLÈME DE LA DATATION}

Le matériel découvert lors de l'intervention concerne surtout les domaines de la construction et de la décoration. Sans entrer dans le détail, il nous parait important de l'évoquer car il peut jouer un rôle dans l'interprétation de la fonction de l'établissement.

La vaisselle céramique est rare puisque seulement 41 fragments ont été recueillis. Le seul élément d'importation est un récipient en sigillée Walter 79/80 de Gaule centrale. Cette pauvreté se traduit concrètement par l'impossibilité d'élaborer une chronologie absolue. L'anse en alliage cuivreux découverte dans l'US 1001 (fig. 5) ne fournit aucune indication et le monnayage comprend uniquement un denier en argent de Commode, découvert fortuitement. Les terres cuites architecturales sont à l'inverse très nombreuses. Ont été prélevés en divers points du site plusieurs fragments de tubuli, des pilettes d'hypocauste ainsi que des dalles de maçonnerie employées lors de la construction de suspensurae. À ces artefacts s'ajoutent de très nombreux morceaux de dalles de schiste, de plinthes ou de réglettes.

L'élément le plus remarquable est un fragment de plaque murale sculptée figurant un pilastre (fig. 12), qui est à rapprocher d'une découverte réalisée sur la villa de Châtillonsur-Seiche en Ille-et-Vilaine (Langouët et Quesnel, 2001). De telles plaques sont signalées généralement dans des édifices thermaux et c'est également à ce type de construction que renvoient les terres cuites architecturales. Tous ces objets sont ici en réemploi : ils proviennent de remblais, à l'image de deux pilettes encore jointoyées au mortier mises au jour dans le remplissage du fossé drainant 1044, daté de la première phase. La présence de tubuli dans la mise en œuvre de l'évacuation de la salle 6 et dans celle du sol de la pièce 7 illustre ce phénomène de récupération tout en fournissant un indice chronologique bien maigre puisque leur apparition semble se produire durant la première moitié du $\mathrm{I}^{\mathrm{er}}$ siècle de notre ère en Italie (Adam, 1989, p. 293).

Ces artefacts ne conduisent nullement à une perception chronologique satisfaisante du fonctionnement du bâtiment ou de ses transformations. La coupe en sigillée Walter 79/80 et le denier de Commode indiquent qu'il devait être encore en fonction à la charnière des $\mathrm{II}^{\mathrm{e}}$ et $\mathrm{III}^{\mathrm{e}}$ siècles de notre ère. Les plaques murales sculptées se rencontrent durant toute la période romaine mais sont surtout signalées dans des bâtiments construits ou réaménagés au cours des $\mathrm{II}^{\mathrm{e}}$ et III ${ }^{\mathrm{e}}$ siècles (Langouët et Quesnel, 2001). L'utilisation de nombreux éléments de bains dans cette construction encourage en tout état de cause une datation tardive puisqu'ils procèdent $\mathrm{du}$ démantèlement d'un bâtiment pré-existant.

\section{INTERPRÉTATION DE L'ÉDIFICE}

\section{Les caractéristiques générales du bâtiment}

Outre un problème de chronologie, la principale question est de saisir la fonction de l'édifice car, si son plan apparaît assez simple au premier abord, sa conception et sa destination restent d'appréhension délicate, en raison de son arasement et du manque d'indices concrets. Même si les données sont parfois lacunaires, notamment pour la phase 1, l'édifice a dû être prévu dès sa construction pour une fonction bien particulière, qui s'est maintenue durant toute la durée de son occupation. Malgré des extensions progressives ou des rénovations, les anciens espaces étaient en effet toujours conservés.

L'environnement a aussi conditionné la méthode de construction. La position topographique du bâtiment est propice à un fort ruissellement, tandis que l'existence de sources et d'un ruisseau témoigne de l'humidité du secteur. Ces conditions expliquent la réalisation d'un système fossoyé le long de certaines maçonneries lors des deux premières phases, afin de drainer l'eau du sous-sol vers le talweg. Elles se ressentent aussi dans la mise en œuvre des murs : la maçonnerie 2015 repose sur une assise de quartz, au même titre que M 2042. Un constat similaire peut être avancé pour les murs des pièces 1 et 2 . Le choix de ce matériau dans la réalisation des murs du canal n'est pas anodin mais résulte certainement de sa résistance à l'eau. On a par ailleurs pris soin de ne pas utiliser le granite en parement mais plutôt le micaschiste, le premier ayant tendance à s'altérer rapidement dans un milieu humide. Cette volonté de protéger l'édifice des remontées d'eau par capillarité se discerne également au travers de la conception du radier et du rudus de la salle 6 et de l'étalement de remblais drainants.

L'eau est au centre de la fonction de la construction. Un bassin est avéré à la phase 2 avec la pièce 6 et le revêtement de mortier de tuileau observé sur le parement est du mur 2003 pourrait souligner que la salle 1 est également un bassin. À la phase 3, la salle 7 correspond aussi à une structure en eau. L'hypothèse de cuves disposées en cascade (trois lors 


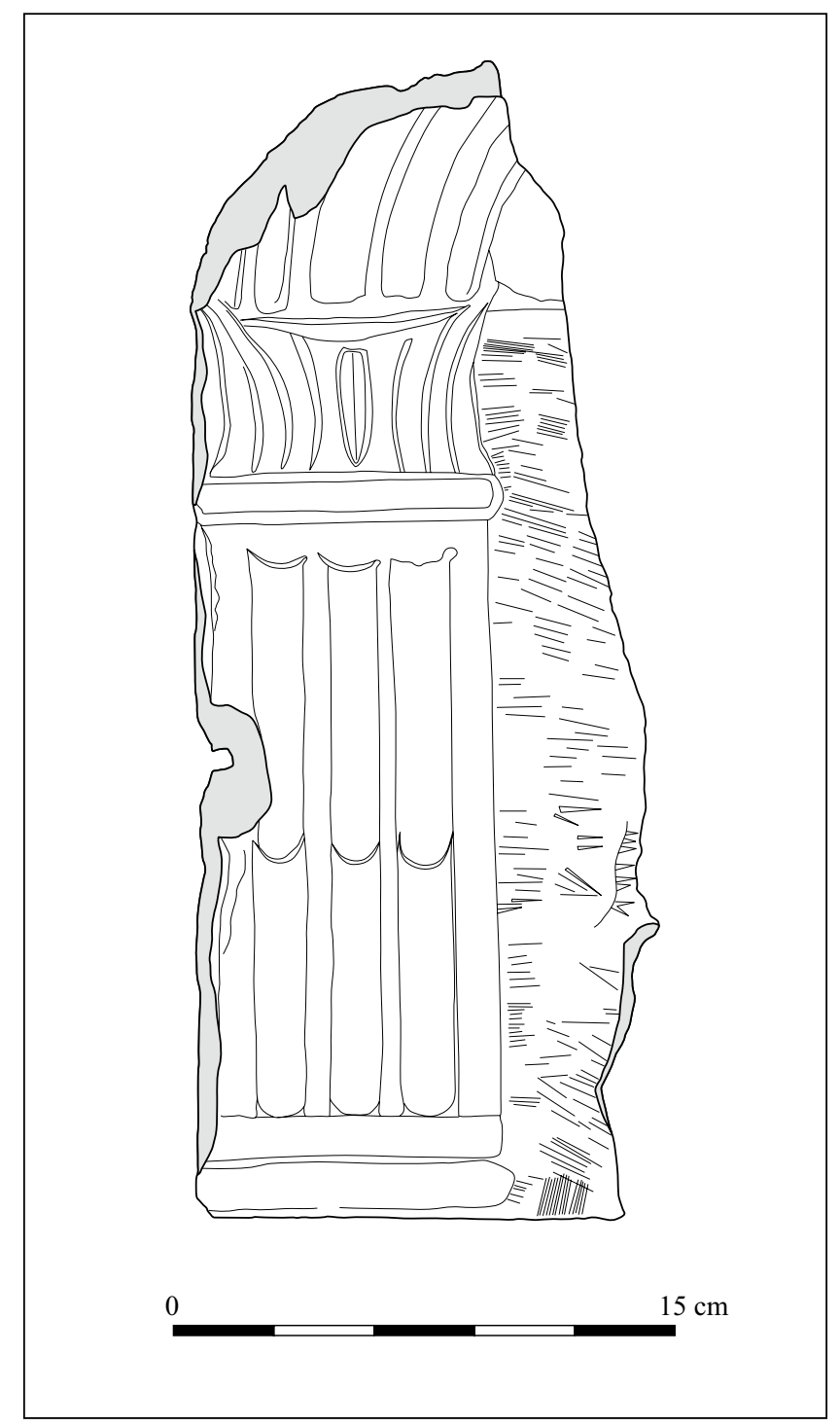

Figure 12 : Plaque murale sculptée en schiste trouvée dans un remblai (DAO S. Jean - INRAP).

Figure 12: Sculptured schist wall slab, from a backfill.

des phases 2 et 3) est plus que plausible. Cette supposition est validée par le cheminement du canal 2023, qui ceinture une bonne partie de la construction lors des deux dernières phases. À son sujet, il est fort probable que les terminaisons des murs 2011 et 2038 ne soient qu'artificielles et dues à l'érosion. On peut alors s'interroger sur l'existence à proximité du bâtiment étudié d'aménagements non conservés, dont la vidange se serait également faite dans 2023. En outre, la largeur et la mise en œuvre très élaborée de ce canal d'évacuation sont dignes de certains égouts urbains ${ }^{2}$, voire

2. Pour exemple, citons les égouts d'Amiens, dont la mise en œuvre est similaire (Bayard et Massy, 1983, p. 112-113). d'aqueducs ${ }^{3}$. Assurément, l'activité pratiquée nécessitait un apport d'eau conséquent, dont nous ignorons l'origine, mais dont témoigne l'évacuation 2023.

\section{La définition d'espaces et le problème de la circulation}

Lors de son extension maximale, l'édifice de "La Mézeray " se décompose en quatre espaces fonctionnels distincts. Le premier comprend les bassins 7, 1 et 2 ainsi que l'évacuation 2023. Le second correspond à la pièce 4, qui se présente comme un espace non cloisonné d'une superficie inférieure à $30 \mathrm{~m}^{2}$. La salle 3 constitue une entité à part entière, mais qui pourrait être liée à la précédente. Enfin, la fonction de la pièce 6 , qui n'est désormais plus un bassin, et celle de la salle 8 sont difficilement déterminables. Cette dernière peut toutefois faire office d'accès.

Lors de la deuxième phase, le sol de la pièce 6 devait en outre surplomber ceux des bassins 1 et 2 . En revanche, nous ignorons si une différence de niveaux existait entre ces derniers, du fait des récupérations de matériaux opérées. En outre, et ce quelle que soit l'époque, les niveaux d'utilisation des salles 4 et 3 ne pouvaient que se trouver à une altitude supérieure à ceux des pièces 1 et 2 d'après le mode de construction mis en œuvre, puisque le sol naturel n'a fait l'objet d'aucun décaissement à leur emplacement. Cette différence altimétrique justifie la largeur de $80 \mathrm{~cm}$ des murs 2026, 2032, 2034 et 2043, afin de contenir les terres ou remblais situés au niveau des salles 3 et 4 . Le mur 2030 qui les sépare est un simple cloisonnement de l'espace en avant des bassins, espace qui devait disposer de sols situés à la même altitude. Lélévation des salles 3 et 4 et leur couverture pouvaient aussi nécessiter des maçonneries puissantes afin de maintenir la cohésion de l'ensemble. À l'inverse, les élévations des salles 1, 2 et 7 devaient être plus modestes, à en juger d'après la largeur moindre des murs. Du point de vue de la circulation, il est certain que l'accès aux salles 7 et 2 devait se faire depuis la salle 4 . L'accès à la salle 1 était possible depuis la pièce 3 . Ceci implique une communication assez simple, nord-sud, se traduisant par des ouvertures dont il est difficile de restituer les dimensions. Toutefois, au niveau de la salle 7 , le redent observé dans le mur 2034, à la hauteur du sol 2027, pourrait en matérialiser l'accès, qui serait alors de $2 \mathrm{~m}$ de large.

\section{Un bâtiment artisanal?}

Parmi les constructions nécessitant de l'eau, les thermes sont les monuments les plus communs et documentés. La

3. Citons, pour la Bretagne, l'aqueduc de Carhaix, dont le canal de circulation d'eau est large de $80 \mathrm{~cm}$ (Provost, 1999). 
probabilité de bains privés, déconnectés physiquement de l'habitat comme il s'en rencontre en Bretagne (Le Bot, 2003) est cependant à exclure ici. Malgré la découverte de nombreux artefacts les concernant, le plan de l'établissement ne correspond pas à leur agencement, notamment du point de vue de la circulation entre les différents espaces. Aucun praefurnium n'a été mis en évidence et la configuration du bâtiment lors de sa dernière phase laisse perplexe quant à sa localisation éventuelle. Les tubuli, pilettes et autres dalles de schiste recueillis procèdent, comme nous l'avons déjà indiqué, du démantèlement ou de la rénovation d'un édifice proche, dont les matériaux ont ensuite été réutilisés.

L'hypothèse d'un nymphée ou d'une fontaine est aussi à abandonner car l'eau n'est pas mise en valeur (Gros, 2002, p. 420) mais occupe au contraire un rôle utilitaire au sein de la construction. D'autres édifices consommateurs d'eau en grande quantité concernent par contre le domaine de l'artisanat, notamment le travail des étoffes ou du cuir, à l'image des fullonicae, des teintureries, ou des tanneries.

Le travail du cuir se décompose en trois temps successifs, qui impliquent un circuit précis et une division tripartite des ateliers : le "travail de rivière " qui peut comprendre quatre étapes, le tannage et enfin le corroyage (Leguilloux, 2004). Le " travail de rivière " vise à préparer les peaux avant le tannage proprement dit. Les finitions, ou corroyage, sont destinées à assouplir et à lustrer le cuir. Ces deux phases ne réclament pas d'installations lourdes et complexes, la première pouvant être réalisée dans de simples fosses. En revanche, le tannage, qui nécessite plusieurs bains afin d'obtenir un cuir de bonne qualité, pouvait se pratiquer dans des cuves maçonnées et cylindriques, à l'image de l'officina coriara de Pompéi qui en comptait une quinzaine à sa période de plein fonctionnement (Leguilloux, 2002). Ces structures sont parfois beaucoup plus légères comme l'illustre la tannerie de la Clinique Richelieu à Saintes (Hillairet, 1995, p. 139). À Liberchies en Belgique, le tannage s'effectuait dans de simples cuves en bois (Brulet et al., 2001; Dewert, 1995). Ajoutons qu'il n'est pas rare de retrouver de nombreux éclats d'os provenant du nettoyage des peaux, lorsque ces dernières sont achetées en ville auprès des bouchers (Leguilloux, 2004, p. 156). Sur le site de « La Mézeray ", la faune est quasi absente, mais l'hypothèse d'un rejet des déchets aux abords de la construction, ou la disparition de ceux-ci en raison de l'acidité du sol, reste plausible. À la lecture de son plan et en raison de sa conception, l'établissement de Plouër-sur-Rance peut toutefois difficilement correspondre à une telle installation.

Autre activité artisanale utilisant l'eau, la teinture pouvait concerner des fils en écheveaux mais aussi des pièces déjà tissées. Dans ce cas, les étoffes étaient teintes a posteriori et de couleur unie (Ferdière, 1984 p. 228; Roche-Bernard et Ferdière, 1989 p. 103). L'une des caractéristiques essentiel- les d'une teinturerie est l'existence de chaudières, comme le montrent les exemples de Pompéi. Elles se justifient par l'emploi de colorants à mordants afin de fixer la couleur des textiles, qui demandent à être chauffés à haute température. Dans ce cas, le mordant utilisé pouvait être de l'urine ou des cendres. L'emploi de l'alun est aussi attesté par les textes et par la découverte d'amphores de Lipari dans deux officinae coriarae de Pompéi. Des bassins sont présents mais paraissent voués à un usage secondaire. Ainsi, ceux de l'officine V 1, 4 ne disposent ni d'adduction ni d'évacuation et sont construits alors que l'atelier est déjà en activité (Borgard, 2002).

Sur le site de "La Mezeray ", l'absence de chaudières peut se comprendre par l'état dégradé du site. Toutefois, aucun fragment d'amphore de Lipari n'est recensé, alors même qu'elles sont très présentes à Corseul (Borgard, 2001) ${ }^{4}$. L'existence de plusieurs bassins ne semble pas non plus en adéquation avec ce type d'officine.

Un second procédé, appelé teinture à froid, nécessite des équipements beaucoup plus légers, des cuves, qui peuvent être maçonnées, en bois, voire de simples jarres. Il implique le trempage des fibres dans un bain où ont été mis à macérer dans de l'eau tiède des colorants naturels. Plusieurs trempages entrecoupés d'expositions à l'air sont nécessaires, expliquant la disposition en série des cuves. C'est sans doute ce type de procédé qui a prévalu dans l'officine découverte à Barcelone, où la présence d'indigo a été mise en évidence dans l'un des bassins (Beltran de Heredia Bercero et Jordi y Tresseras, 2000). En Gaule, le seul exemple connu demeure Saint-Romain-en-Gal, où deux cuves rectangulaires enduites de tuileau ont été assimilées à cet artisanat en raison de la découverte de pigments dans des restes de jarres. L'interprétation reste incertaine et la possibilité d'une petite fullonica a été avancée (Leblanc, 1996). Concernant le site de "La Mézeray ", les vestiges sont trop arasés pour y reconnaître une teinturerie utilisant ce procédé.

Une fois tissées, les pièces subissent plusieurs opérations avant d'être commercialisées. Celles-ci interviennent dans les fullonicae, dont les installations sont surtout connues grâce aux fouilles de Pompéi ou d'Ostie (De Ruyt, 2001; 2002) ${ }^{5}$. Plusieurs inscriptions ou documents iconographiques concernent également la Gaule (Ferdière, 1984; Pelletier, 1996). Le travail se décompose en trois étapes principales. Des ouvriers piétinent d'abord les tissus, qui trempent dans une solution à base d'urine et d'argile, dite terre à foulon. Cette opération, qui permet de resserrer la trame et de dégraisser les étoffes, se pratique dans des bacs appelés fouloirs. Ce sont des récipients

4. Rappelons que l'alun pouvait être aussi utilisé dans des ateliers de mégisserie comme agent tannant ou comme mordant pour teindre les cuirs (Le Guilloux, 2004, p. 31-33, Borgard et al., 2002).

5. Nous ne traitons pas ici de la question des blanchisseries, destinées au nettoyage des tissus en usage. 
dont le nombre et la nature sont variables en fonction des établissements. Généralement, ils sont accolés contre un mur et séparés par des murets ou parapets sur lesquels prennent appui les ouvriers. À Pompéi, deux cuvettes ovales, de $30 \mathrm{~cm}$ de profondeur, dont le sommet coïncide avec le sol de circulation, ont été identifiées comme des fouloirs dans l'officine I 6, 7 (Borgard et Puybaret, 2005). À Ostie, de petites cuves côtoyaient des amphores récupérées et retaillées dans l'atelier III ii 2 (De Ruyt, 2001). En somme, ce sont des installations assez sommaires. Les étoffes sont ensuite rincées à l'eau claire afin d'être purifiées. Cette opération s'effectue dans des bassins maçonnés creusés à même le sol et situés à proximité des bacs à foulons. À Pompéi, les principaux établissements, installés dans d'anciennes domus, comprennent généralement trois bassins, dont un seul est alimenté par le réseau public, le remplissage des deux autres se faisant grâce au principe des vases communicants. Leur capacité est variable, toujours supérieure à 2500 litres, mais le bassin relié au système d'adduction est toujours de capacité plus importante et pourrait constituer une réserve (Borgard et Puybaret, 2001). La même disposition semble se retrouver dans les officines d'Ostie, dont certaines peuvent contenir quatre bassins (De Ruyt, 2001) : alimentation des cuves à partir d'un bassin-fontaine relié au réseau public. La dernière opération regroupait les finitions : battage, séchage à l'abri du soleil, brossage... Ces trois étapes font appel à un circuit précis, donc à des espaces spécifiques. Le plan de l'édifice de Plouër-sur-Rance peut-il s'apparenter à ce type d'activité?

Aucun bac de fouloir n'a été retrouvé lors de l'intervention. Une explication plausible tient dans l'arasement du site. Aucun sol n'est conservé au nord des pièces 1 et 2 . Or, dans l'hypothèse d'une fullonica, leur emplacement est sans doute à rechercher dans ce secteur du bâtiment, peut-être le long de M 1030 ou de M 1015. Les salles 6, 1, 2 et 7 pourraient correspondre alors à des bassins de rinçage, situés à proximité des bacs. On peut penser qu'un parapet devait les isoler de toute souillure. Ces quatre bassins n'ont pourtant pas fonctionné de façon synchrone. Durant la phase 1, seuls les numéros 1 et 2 sont assurés. Lors de la deuxième phase s'adjoint la salle 6. L'ultime phase marque la condamnation de cette dernière et la création d'un nouvel espace, qui toutefois est de plan très différent (salle 7). Lors des deux dernières périodes, on retrouve le même nombre de bassin qu'à Pompéi. Malheureusement, nous ne pouvons affirmer que la salle 6 soit une sorte de réserve. Son système d'alimentation n'est pas assuré, au même titre que l'adduction des bassins 1 et 2 . Rien n'interdit cependant d'imaginer une alimentation par trop-plein de ces dernières, l'édifice étant semble-t-il construit en escalier (fig. 13).

Au sortir du rinçage, les tissus pourraient être travaillés dans l'espace au nord des bassins, au niveau de la salle 4, la plus grande en superficie. La salle 3 abriterait alors les bacs de foulons. En plan, rien ne semble s'opposer à une officina fullonica. Les vestiges mobiliers que ce type d'activité engendre sont finalement peu nombreux, hormis les matières organiques périssables ou des outils en fer telles que des forces. L'absence de fouloirs laisse néanmoins planer une incertitude.

En Gaule, plusieurs fouleries sont identifiées ou du moins pressenties. L'exemple le plus célèbre demeure Saint-Romainen-Gal (Leblanc, 1996; Brissaud, 1999). Trois autres établissements sont aussi attestés en Narbonnaise, deux à Fréjus (Rivet et al., 2000, p. 438-439) et un dernier à Nîmes, sur le site du Clos-de-la-Lombarde (Sabrié et al., 2001, p. 122125). En Gaule Lyonnaise, aucun édifice certain n'est connu mais deux sont présumés dans la région Centre à Amboise et à Orléans (Ferdière, 1984, p. 229) et un troisième près de Creil, sur l'agglomération de Litanobriga (Roche-Bernard et Ferdière, 1993, p. 121). Une partie d'une officine a sans doute été abordée à Limoges (Loustaud, 2000, p. 268-269). Pour la région Bretagne, $\mathrm{P}$. Galliou mentionne, en tant qu' installation de foulons, une cuve rectangulaire découverte près des thermes de Roz-Avel en Quimper (Galliou, 2005, p. 226) ${ }^{6}$.

À travers ces quelques cas, cet artisanat semble l'apanage des agglomérations et des villes, ce que tendent à démontrer la littérature et les découvertes archéologiques, notamment épigraphiques (Pelletier, 1996). Les exemples cités viennent donc fragiliser l'interprétation en ce sens du bâtiment de "La Mézeray ", même si certains sites évoqués pourraient être de simples blanchisseries.

Ici, l'agglomération la plus proche est en effet Taden, distante de près de $10 \mathrm{~km}$. Pour autant, l'établissement de "La Mézeray " n'est pas totalement isolé puisque trois sites antiques sont répertoriés dans sa périphérie. Deux sont localisés à moins de $200 \mathrm{~m}$ et le dernier à un peu plus de $400 \mathrm{~m}$. La nature et la chronologie de ces gisements, repérés lors de prospections pédestres, demeurent inconnues. Ils témoignent de l'attrait du secteur, grâce à l'omniprésence de l'eau ou la qualité des terres agricoles. On peut en tout cas, au vu de leur proximité, s'interroger sur l'intégration de la construction étudiée ici dans un ensemble beaucoup plus vaste. Quelle que soit la fonction exacte de l'édifice, il est en effet difficile de croire à son isolement total, puisqu'il ne s'agit pas d'un habitat. La découverte de nombreux éléments de bains conforte cette proposition car on imagine mal leur transport sur une longue distance. Peut-être cet édifice appartient-il à un domaine foncier proche? Sa fonction ne traduirait-elle pas alors une partie de la production de ce domaine? Quelques cas de foulerie en milieu rural sont

6. Précisons que cette liste ne prétend aucunement à l'exhaustivité. 


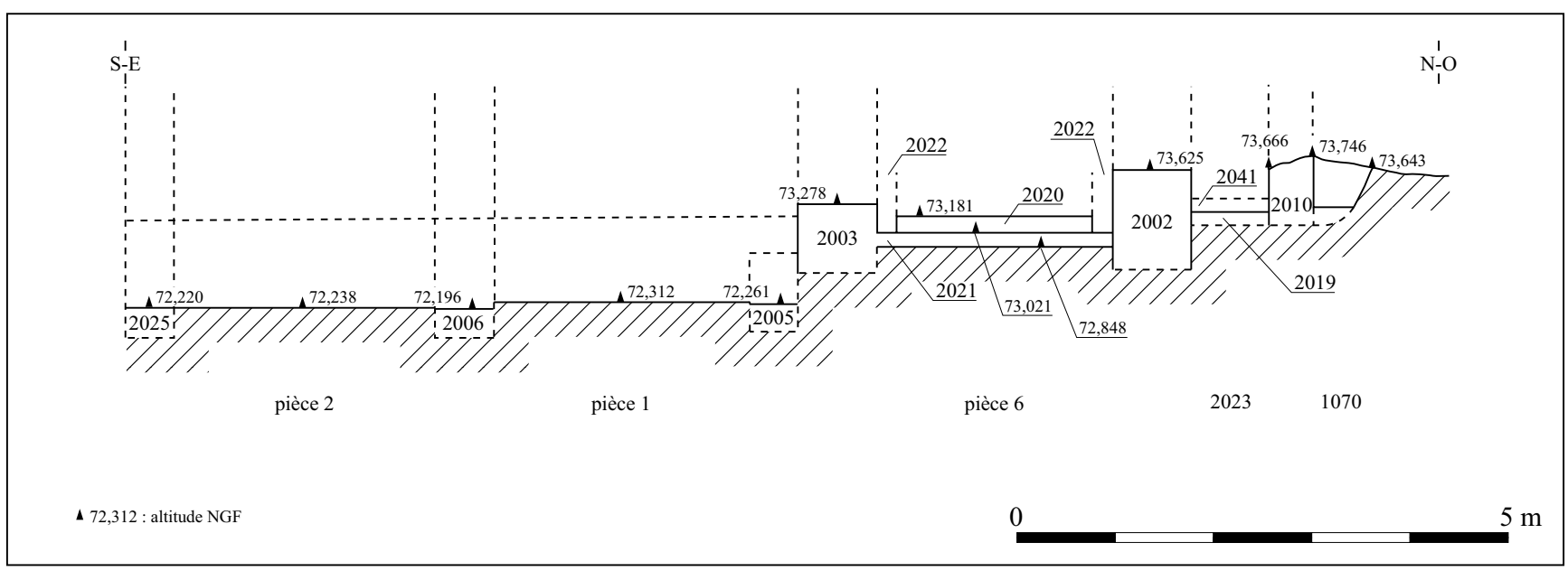

Figure 13 : Schéma montrant la disposition étagée de la canalisation 2023 et des pièces 6, 1 et 2 lors de la phase 2 (DAO S. Jean - INRAP).

Figure 13: Sketch of the stepped layout of ditch 2023 and rooms 6, 1 and 2 during phase 2.

signalés par J.-C. Béal (2002), notamment celle de Caton l'Ancien, mais cet exemple est à manier avec précaution car il concerne un haut personnage public de la République romaine et le modèle est difficilement transposable. Des bassins maçonnés, localisés à $1,5 \mathrm{~km}$ de la ville de Lillebonne (Seine-Maritime) et découverts au début du Xx $x^{e}$ siècle dans un fond de vallée, sont aussi interprétés comme des installations de foulons (Rogeret 1997, p. 376). Plus récemment, la fouille d'une villa réalisée sur le tracé de l'autoroute A 75 a révélé l'existence de deux cuves qui ont pu servir au traitement de la laine tout en posant la question de la destination de cette production (Mauné 2003).

Il convient dès lors de s'interroger sur la portée de cette proposition, car elle implique l'existence d'une exploitation foncière intégrant l'édifice de "La Mézeray " et dont la production serait axée sur l'élevage ovin ou sur le lin. Or aucune donnée concrète ne vient étayer cette suggestion. Les informations sur les sites répertoriés à proximité sont trop succinctes pour y voir l'existence d'une villa: les indices se résument à de la céramique associée à des tegulae et des éléments d'hypocauste comme à "La Houssaye " (Langouët et Daire, 1989). Néanmoins, L. Langouët et G. Jumel (1991) considèrent, à partir de la répartition des pesons de tisserands, que les zones granitiques de la civitas des Coriosolites sont propices à l'élevage du mouton. Un exemple un peu plus éloigné, situé chez les Riedones, semble aller dans ce sens : l'étude de la faune de la villa de Châtillon-sur-Seiche, au sud de Rennes, y a mis en exergue l'importance de l'élevage d'ovicaprins au cours du Haut-Empire avec 20 à $25 \%$ des restes osseux (Provost, 1990, p. 40).

Admettre que le bâtiment de "La Mézeray " est une fullonica revient à dire que l'établissement dont il dépendait, quelle que soit sa nature, contrôlait aussi les étapes nécessaires en amont du foulage. Une fois tondue, la laine doit subir une première préparation afin d'être dégraissée et débarrassée de ses impuretés, et enfin cardée. Ensuite seulement interviennent le filage puis le tissage. Tout ceci demande une parfaite organisation et surtout du personnel, ne serait-ce que pour le filage, particulièrement long et considéré comme une activité féminine (Roche-Bernard et Ferdière, 1993, p. 7172). Or, si le filage est une opération relevant des exploitations agricoles, le tissage est souvent perçu, grâce aux cartes de répartition des pesons de tisserands, comme une tâche réalisée dans les villes et les habitats groupés. Encore faut-il préciser que ces objets ne sont pas toujours le reflet d'un travail de la laine mais peuvent aussi concerner la mise en œuvre de plantes textiles, dont le lin. Une certaine prudence doit donc être de mise quant à leur découverte et aux interprétations qui en découlent.

Dans le nord de La Gaule, J.-C . Béal (1996), en confrontant les sources littéraires et les témoignages archéologiques, conclut que l'activité de transformation de la laine serait très structurée et concentrée dans les villes. Pour le Val-de-Loire, la production textile paraît être le fait des agglomérations (Provost, 1993, p. 254), ce qui serait aussi le cas dans le Centre de la France (Ferdière, 1984, p. 225). Une situation différente se dessine pour l'ouest et le sud-ouest, où l'activité textile serait du ressort de grandes villae (Béal, 1996). Concernant la cité des Coriosolites, les pesons sont surtout attestés sur des établissements ruraux (Ferdière, 1989; Langouët et Jumel, 1986) et, pour les Côtes-d'Armor, sur les sites du nord du département (Bizien-Jaglin et al., 2002, p. 50). Cette image, qui est le reflet pour partie des prospections, est sans doute à nuancer puisque l'opération de 
Monterfil II à Corseul a livré une vingtaine de pesons, qui soulignent aussi une pratique urbaine du tissage ${ }^{7}$.

\section{Conclusion}

La fouille du site de "La Mézeray " a livré un établissement au plan relativement simple mais évolutif et à la fonction difficilement déterminable. Nous avons essayé de démontrer dans les lignes précédentes que son installation en un lieu a priori peu propice n'était pas le fruit du hasard mais répondait à un besoin bien particulier, qui nécessitait un apport d'eau conséquent. Même si les indices sont maigres, cet établissement ne peut être isolé et doit s'intégrer dans un ensemble plus vaste, peut-être une villa. Son plan, qui ne correspond pas à un habitat - et l'hypothèse de thermes privés étant à exclure - argumente en ce sens. Dans ces conditions, il faut admettre qu'une activité spécifique y était prévue dès l'origine, ce que ne dément pas l'analyse du phasage. La possibilité d'un bâtiment reflétant la production d'un domaine foncier n'est sans doute pas à remettre en cause; seule sa finalité exacte, qui utilise en abondance l'eau, reste problématique. L'hypothèse d'un atelier de traitement de la laine est une des possibilités mais elle ne peut être démontrée au seul examen de vestiges archéologiques fortement arasés.

Si notre hypothèse s'avérait exacte, quels seraient alors la capacité de production et les débouchés de cet établissement? Alimentait-il les exploitations rurales ou les agglomérations de Taden, d'Alet? Sa production était-elle aussi destinée à Corseul où une importante activité de teinturerie est pressentie, certes pour le premier siècle de notre ère? Plus de trois mille tessons d'amphores de Lipari ont en effet été découverts sur le site de Monterfil II (Borgard 2001). Doit-on alors envisager une redistribution d'une partie de la production textile de l'édifice de "La Mézeray " vers cette cité, soit pour y être teinte, et dans ce cas les textiles seraient monochromes, soit pour y être directement vendue? Ces questions demeurent sans réponse mais cet établissement pourrait constituer un exemple des liens entre une ville et son milieu rural. À ce titre, cette suggestion rejoindrait l'idée qu'une bonne partie de la production artisanale est le fruit de domaines ruraux aux mains de l'élite des cités (Ferdière 1999; 2005).

7. Donnée inédite.

\section{Bibliographie}

AdAM, J.-P., 1989 - La construction romaine. Matériaux et techniques, Paris, Picard, 368 p.

Aubry, L., Ferrette, R., Gaumé, E., Paitier, H., Jean, S. et Pommier, V., 2002 - Plouër-sur-Rance "La Mézeray " Côtesd'Armor, Document final de synthèse, Rennes, SRA de Bretagne.

BAyARD, D. et Massy, J.-L., 1983 - Amiens romain (Samarobriva Ambianorum), Revue archéologique de Picardie ( $\mathrm{n}^{\circ}$ spécial), $374 \mathrm{p}$.

BÉAL, J.-C., 1996 - Instrumentum et production textile en Gaule romaine : l'exemple des pesons de terre cuite. Aspects de l'artisanat du textile dans le monde méditerranéen (Égypte, Grèce, Monde Romain), Lyon, Université Louis-Lumière - Lyon 2, coll. «Institut d'Archéologie et d'Histoire de l'Antiquité, vol. $2 »$, p. 121-132.

BÉAL, J.-C., 2002 - L'artisanat et la ville romaine : relecture de quelques textes, in BÉAL, J.-C. et GuYon, J.-C. (dir.), Les Artisans dans la ville antique, Lyon, Université Louis-Lumière - Lyon 2, coll. "Archéologie et Histoire de l'Antiquité », p. 5-14.

Beltran de Heredia Bercero, J. et Jordi y Tresserras J., 2000 Resultados de las investigaciones arqueológicas y arqueométricas en las instalaciones de la colonia de Barcino (Barcelona, España), in Cardon, D. et Feugère, M., Archéologie des textiles des origines au ve siècle, Actes du colloque de Lattes, 1999, Montagnac, éditions Monique Mergoil, p. 241-246.

Bizien-Jaglin, C., Galliou, P. et Kerebel, H., 2002 - Côtes-d'A $r$ mor. 22, Paris, Académie des Inscriptions et Belles-Lettres, coll. "Carte Archéologique de la Gaule », 406 p.

Borgard, P., 2001 - "Une agglomération importatrice d'alun? ", in Kerébel, H. (dir.), Corseul (Côtes-d'Armor), un quartier de la ville antique, Paris, MSH, coll. « Documents d'Archéologie Française, 88 ", p. 163-164.

Borgard, P., 2002 - À propos des teintureries de Pompéi : l'exemple de l'officina infectoria V 1, 4, in BÉAL, J.-C. et GuYon, J.-C. (dir.), Les Artisans dans la ville antique. Lyon, Université Louis-Lumière - Lyon 2, coll. "Archéologie et Histoire de l'Antiquité ", p. 55-68.

Borgard, P. et Puybarret, M.-P., 2005 - Approche archéologique du travail de la laine au ${ }^{\text {er }}$ siècle après J.-C. Produzione, Merci e commerci in altino preromana e romana, atti del convegno (Venezia 12-14 dicembre 2001), Studi e Ricerche Sulla Gallia Cisalpina, 17, 299-318.

Borgard, P., Forest, V., Bioul-Pelletier, C. et Pelletier, L., 2002 - "Passer les peaux en blanc": une pratique galloromaine? L'apport du site de Sainte-Anne à Dijon (Côted'Or), in Audoin-Rouzeau, F. et Beylnes, S., Le travail du cuir de la Préhistoire à nos jours, XXII ${ }^{\mathrm{e}}$ rencontres internationales d'archéologie et d'histoire d'Antibes, Antibes, éditions APDCA, p. 231-249. 
Brissaud, L., 1999 - Un vase de foulon sur le site de Saint-Romainen-Gal? Hypothèses et pistes de recherches, in Ballet, P., Cordier P. et Dieudonné-GLAD, N. (dir.), La ville et ses déchets dans le monde romain, Actes du colloque de Poitiers, Montagnac, éditions Monique Mergoil, p. 61-72.

Brulet, R., Dewert, J.-P. et Vilvorder, F., 2001 - Liberchies IV. Vicus gallo-romain, Louvain-La-Neuve, Publications d'Histoire de l'Art et d'Archéologie de l'Université de Louvain, 438 p.

DE RUYT, C., 2001 - Les foulons, artisans des textiles et blanchisseurs, in Descoeudres, J.-P. (dir.), Ostia, port et porte de la Rome antique, Genève, Musée Rath, p. 186-191.

De RuYT, C., 2002 - Boulangers et foulons d'Ostie à l'époque impériale : quelques réflexions sur l'implantation de leurs ateliers et sur leurs fonctions précises dans la ville portuaire, in BÉAl, J.-C. et Guyon, J.-C. (dir.), Les Artisans dans la ville antique. Lyon, Université Louis-Lumière - Lyon 2, coll. "Archéologie et Histoire de l'Antiquité », p. 49-54.

Dewert, J.-P., 1995 - Liberchies (Pont-à-Celles) : "Les Bons Villers ». Un complexe artisanal imposant: une seconde tannerie, Mons (Hainaut), Ministère de la région Wallonne/DGATLP, p. 17-18.

Ferdière, A., 1984 - Le travail du textile en région Centre de l'âge du Fer au haut Moyen-Âge. Revue archéologique du Centre de la France, 23, p. 209-275.

Ferdière, A., 1989 - Économie rurale et production textile en Gaule romaine (Belgique, Lyonnaise, Aquitaine), Tissage, corderie, vannerie, Juan-les-Pins, APDCA/Centre de Recherches archéologiques du CNRS, p. 181-191.

FERDIÈRE, A., 1999 - L'artisanat gallo-romain entre ville et campagne (histoire et archéologie) : position historique du problème, méthodologie, historiographie, in Polfer, M. (dir.), Artisanat et productions artisanales en milieu rural dans les provinces $d u$ nord-ouest de l'Empire romain, Montagnac, éditions Monique Mergoil, p. 9-24.

Ferdière, A., 2003 - La place du domaine foncier dans la production artisanale destinée au marché. Cultivateurs, éleveurs et artisans dans les campagnes de Gaule romaine : matières premières et produits transformés (Actes $\mathrm{du} \mathrm{VI}^{\mathrm{e}}$ colloque de l'association AGER), Revue archéologique de Picardie, $\mathrm{n}^{\circ}$ 1-2, p. 263-274.

Ferdière, A., 2005 - L'artisanat en Gaule romaine, in Rivet, L. (dir.), Spécificité et diffusion de la céramique gallo-romaine en région Centre. Actualité des recherches céramiques (Actes du congrès de Blois), Marseille, Société française d'études de la Céramique antique en Gaule, p. 7-14.

Galliou, P., 2005 - L'Armorique romaine, Brest, Armeline, 415 p.

Gros, P., 2002 - L'architecture romaine. 1 - Les monuments publics, Paris, Picard, coll. « Les Manuels d'Art et d'Archéologie antiques, $2^{\mathrm{e}}$ édition », $503 \mathrm{p}$.

Hillairet, J.-L., 1995 - Artisanat antique à Saintes. Saintes, Société d'Archéologie et d'Histoire de la Charente-Maritime, coll. « Recherches Archéologiques en Saintonge », 175 p.
LANGOUËT, L., 1985 - Un vicus gallo-romain, routier et portuaire : Taden (Côtes-du-Nord), sur les bords de la Rance, Revue archéologique de l'Ouest, 2, p. 73-82.

LANGouËt, L., 2004 - Le vicus gallo-romain de Taden (Côtesd'Armor), étape routière et port coriosolite, Les Dossiers $d u$ Centre régional d'Archéologie d'Alet, 32, p. 39-52.

LANGOUËT, L. et JumEL, G., 1991 - Les campagnes gallo-romaines de la civitas des Coriosolites, in LAngouëT, L. (dir.), «Terroirs, territoires et campagnes antiques. La prospection archéologique en Haute-Bretagne. Traitement et synthèses des données ", Revue archéologique de l'Ouest, suppl. n 4, p. 127-134.

LANGouëT, L. et DaIRE, M.-Y., 1989 - La civitas gallo-romaine des Coriosolites. Le milieu rural, Saint-Malo, Centre régional d'Archéologie d'Alet, $81 \mathrm{p}$.

LANGouët, L. et Quesnel, L., 2000 - Les plaques décorées en schiste de la Bretagne armoricaine sous l'Empire romain, Revue archéologique de l'Ouest, 17, p. 215-237.

Leblanc, O., 1996 - Les bassins de foulons de Saint-Romainen-Gal (Rhône). Aspects de l'artisanat du textile dans le monde méditerranéen (Égypte, Grèce, Monde Romain). Lyon, Université Louis-Lumière - Lyon 2, coll. « Institut d'Archéologie et d'Histoire de l'Antiquité, vol. 2 ", p. 137-138.

LE Bot, A., 2003 - Les thermes gallo-romains en Armorique. Rennes, Les Dossiers du CeRAA, suppl. Z, 123 p.

Leguilloux, M., 2002 - Techniques et équipements de la tannerie romaine : l'exemple de l'officina coriara de Pompéi, in AudoinRouzeau, F. et Beylnes, S., Le travail du cuir de la Préhistoire à nos jours, $\mathrm{XXII}^{\mathrm{e}}$ rencontres internationales d'archéologie et d'histoire d'Antibes, Antibes, éditions APDCA, p. 267-282.

Leguilloux, M., 2004 - Le cuir et la pelleterie à l'époque romaine. Paris, éditions Errance, coll. "Les Hespérides », 185 p.

Loustaud, J.-P., 2000 - Limoges antique, Limoges, Association des Antiquités historiques du Limousin (Travaux d'Archéologie limousine, suppl. 5), 387 p.

Mauné, S., 2003 - La villa gallo-romaine de « Vareilles » à Paulhan (Hérault; fouille de l'autoroute A 75), un centre domanial du Haut-Empire spécialisé dans la viticulture. Cultivateurs, éleveurs et artisans dans les campagnes de Gaule romaine : matières premières et produits transformés (Actes du VI ${ }^{\mathrm{e}}$ colloque de l'association AGER), Revue archéologique de Picardie, $\mathrm{n}^{\circ}$ 1-2, p. 309-337.

Pelletier, A., 1996 - Les métiers du textile en Gaule d'après les inscriptions. Aspects de l'artisanat du textile dans le monde méditerranéen (Égypte, Grèce, Monde Romain), Lyon, Université Louis-Lumière - Lyon 2, coll. "Institut d'Archéologie et d'Histoire de l'Antiquité, vol. 2 », p. 133-136.

Provost, A., 1990 - Nos ancêtres les Riedones. La villa gallo-romaine de Châtillon-sur-Seiche, Catalogue d'exposition, Rennes, Écomusée du pays de Rennes - La Bintinais, 64 p.

Provost, A., 1999 - L'aqueduc romain de Carhaix. Mémoires d'âme(s). 20 ans de recherches archéologiques en Côtes-d'Armor, 
catalogue d'exposition, Saint-Brieuc, Conseil Général des Côtes-d'Armor, p. 64-67.

Provost, M., 1993 - Le Val de Loire dans l'Antiquité, Paris, CNRS Editions ( $52^{\mathrm{e}}$ supplément à Gallia), $425 \mathrm{p}$.

Rivet, L., Brentchaloff, D., Roucoule, S. et Saulnier, S., 2000 - Atlas topographique des villes de Gaule méridionale. 2Fréjus. Revue archéologique de Narbonnaise, suppl. 32, Travaux du Centre Camille-Jullian, 27, 507 p.

Rogeret, I., 1997 - La Seine-Maritime. 76, Paris, Académie des Inscriptions et Belles-Lettres, coll. "Carte Archéologique de la Gaule », 659 p.
Roche-Bernard, G., et Ferdière, A., 1993 - Costumes et textiles en Gaule romaine, Paris, éditions Errance, coll. "Les Hespérides ", $175 \mathrm{p}$.

Sabrie, M. et SAbrie, R., (dir.), 2001 - Le clos de la Lombarde à Narbonne. Espaces publics et privés du secteur nord-est, Montagnac, éditions Monique Mergoil, 327 p. 